\title{
Altruistic Punishment, Status Conflict, and Knowledge Sharing in the Workplace: An Evolutionary Game Model
}

\author{
Fang Song $\mathbb{D},{ }^{1}$ Xiwu Hu, ${ }^{1}$ Junfeng $\mathrm{Li}^{2}{ }^{2}$ and Michael Allen Watson ${ }^{3}$ \\ ${ }^{1}$ School of Economics and Management, Qinghai Nationalities University, Xining, Qinghai, China \\ ${ }^{2}$ School of Economics, Hebei GEO University, Shijiazhuang, Hebei, China \\ ${ }^{3}$ University of Virginia, Charlottesville, VA 22904, USA \\ Correspondence should be addressed to Fang Song; fangsong@tju.edu.cn
}

Received 12 January 2021; Revised 1 March 2021; Accepted 8 March 2021; Published 12 April 2021

Academic Editor: Polinpapilinho Katina

Copyright (C) 2021 Fang Song et al. This is an open access article distributed under the Creative Commons Attribution License, which permits unrestricted use, distribution, and reproduction in any medium, provided the original work is properly cited.

\begin{abstract}
Second- and third-party altruistic punishments can both improve knowledge sharing, but it is debatable which does so better. We thus concurrently consider the influence of status conflict advantage and disadvantage on knowledge sharing. We also analyze knowledge sharing by introducing an evolutionary game model and conducting simulations, and reveal the following: (1) the appropriate choice for an organization that is increasing its knowledge contribution is third-party punishment; (2) the status conflict advantage and disadvantage play important roles in knowledge sharing. When the status conflict advantage is greater than the disadvantage, the two players eventually share knowledge, and vice versa. Interestingly, when the status conflict advantage is equal to the disadvantage, the player with the stronger sharing inclination has a powerful impact on the player who would rather hoard knowledge, thus ultimately leading him/her to share. (3) Guanxi, knowledge capacity, and synergy revenue have positive effects on knowledge sharing, while the knowledge sharing cost has a negative influence on them. Moreover, the stable equilibrium result is affected by the initial system state. In sum, our results not only promote the understanding of altruistic punishment and status conflict regarding knowledge sharing but also provide practical implications for both organizations and leaders.
\end{abstract}

\section{Introduction}

Knowledge sharing refers to employees sharing their workrelated experiences, expertise, know-how, and contextual information with their peers through informal and formal interactions within or across teams or work units [1]. Knowledge sharing has thus received considerable attention, due to its vital role in the development of organizations' competitive advantages [2]. However, in real life, individuals cannot share their knowledge with others at all times because of knowledge ownership and the need for the protection and pursuit of their own interests. That is, promoting knowledge sharing is a difficult task, as hoarding and guardedly considering knowledge are natural human tendencies, meaning we cannot expect individuals to simply share their ideas and insights because it is the right thing to do [3]. In short, individuals will not share knowledge if they think they can obtain more benefits from hoarding instead of sharing [2].
Consequently, effective incentives can improve knowledge sharing behaviors in the workplace, with extant studies showing that knowledge sharing cannot be forced or mandated but can instead be encouraged and facilitated $[4,5]$. The threat of punishment can effectively induce individuals to share their knowledge, since negative incentives or punishment can be used to reduce free-rider pay-off [6].

Altruistic punishment has thus received considerable attention in academia, as it challenges the basic "homoeconomicus" model of traditional economics. Psychological, economic, and anthropological research shows that subjects have strong tendencies to behave prosocially and are motivated by the personal desires to punish antisocial behaviors at a cost to themselves [7]. That is, not only those whose interests are affected will punish violators (second-party punishment) but also third parties whose interests have not been harmed will also do so (third-party punishment). Such punishment (both second- and third-parties) can effectively 
maintain social norms and is also called altruistic punishment. As effective incentive measures, second- and thirdparty punishments can essentially enhance employees' knowledge sharing behaviors. However, there is no comparative study on the promotion effects of second- and third-party punishments on knowledge sharing. Therefore, we aim to tackle this line of research in the present study.

Individuals' pursuit of status leads to status conflicts, which are attempts to defend or elevate one's own relative status [8]. Prior research on the relationship between status conflict and knowledge sharing shows that knowledge sharing is a behavior that has both benefits and risks. On the one hand, the need for status drives employees to generously share tacit knowledge and special expertise to obtain social recognition and status, as conferred by supervisory appraisal; thus, this can help them achieve sustainable status conflict advantage over other colleagues [9-12]. On the other hand, knowledge sharing can threaten contributors' power and status by reducing their value to the organization and damaging their position, because their expertise and special know-how constitute the source of their current status and employment $[11,13]$. Therefore, knowledge sharing can lead to status conflict disadvantage for contributors. Although existing research can help us understand the relationship between status conflict and knowledge sharing, there is currently no study on the relationship between status conflict and knowledge sharing behaviors that considers status conflict advantage and disadvantage concurrently. We aim to explore this line of research in this study.

To this end, we analyze knowledge sharing behaviors by introducing an evolutionary game model with the following incentive mechanisms: second-party punishment, thirdparty punishment, and mixed second-and-third-party punishment, to determine which punishment mechanism best promotes knowledge sharing within an organization. The model is then used to simultaneously consider status conflict advantage and disadvantage. The stable equilibrium is then obtained by solving the replicator dynamic equations and quantifying the universal dilemma strength. The following issues are investigated by using Matlab for a programming simulation: (1) which among second- and thirdparty punishments is better for improving knowledge sharing and what happens if we adopt the mixed incentive punishment mechanism; (2) how do status conflict advantage and disadvantage influence knowledge sharing behaviors; and (3) how can we provide practical implications for organizations and leaders to encourage knowledge owners to contribute their knowledge? Finally, the practical implications for organizations and leaders are discussed.

The remainder of this paper is organized as follows. Section 2 provides the theoretical foundation by introducing the concepts of altruistic punishment, second- and thirdparty punishments, status conflict, guanxi, and evolutionary game. Section 3 constructs the evolutionary game model by defining variables and the pay-off functions matrix and investigates the evolutionary stable strategy of the model. Section 4 presents the simulation analysis and the results. Section 5 draws conclusions and presents management implications, as well as the research limitations and future research directions.

\section{Relevant Literature}

Prior studies have hitherto contributed to the better understanding of knowledge sharing; however, there are some questions requiring further investigation. For example, as different types of altruistic punishment, second- and thirdparty punishments can both improve knowledge sharing to some extent; nevertheless, which one promotes knowledge contributions better remains debatable. Additionally, sharing knowledge leads to simultaneous individual status conflict advantage and disadvantage, but there are currently no studies analyzing concurrently the knowledge sharing for both sides of the status conflict. Furthermore, most previous works regarding knowledge sharing are empirical and have several limitations. Therefore, it is necessary to investigate knowledge sharing using different research methods. Specifically, we analyze knowledge sharing by introducing an evolutionary game model with the following main factors: second- and third-party punishments and status conflict advantage and disadvantage. The model is then used to simultaneously consider guanxi, professional and general knowledge capacity, and professional and general absorptive capacity. Finally, we conducted a simulation using Matlab.

\subsection{Altruistic Punishment and Knowledge Sharing.} Punishment can effectively reduce free riding. However, punishment is a public good that benefits everyone and, similar to other public goods, is vulnerable to free riding. Those who benefit without paying punishment costs gain more than those who punish. Punishment is thus a topic of wide interest to scholars in the form of altruistic punishment [14], which describes the sacrifice of self-interest to punish the violations of social norms, such as fairness or reciprocity $[15,16]$. This behavior is often modeled using the ultimatum game (UG) [17-19], which illustrates that a sizeable number of people from a wide variety of backgrounds are willing to punish others at a cost to themselves to prevent unfair outcomes or sanction unfair behavior [20]. Among the representative theories used to explain this phenomenon are the fairness preference, strong reciprocity, social preference, social norm activation, emotion theories. Because these punishments can maintain and consolidate social norms, they are called altruistic punishment [20].

The important impact of altruistic punishment is that it challenges the basic hypothesis in traditional economics on the existence of an economically rational agent with two characteristics: it is selfish and pursues the maximization of self-interest instinctively [21]. However, the studies on altruistic punishment indicate that individuals' behaviors can also be irrational and willing to maintain social norms even it means they need to sacrifice their own interests. Such altruistic punishment helps sustain cooperation in society and is present across a highly diverse range of human populations and under various patterns $[22,23]$. 


\subsection{Second-and Third-Party Punishments and Knowledge} Sharing. Altruistic punishment includes second- and thirdparty punishments, both being widely used to punish noncooperators to preserve social norms. Second-party punishment, which is performed by punishers who are directly affected by those whom they punish [24], has attracted the attention of scholars. "An eye for an eye and a tooth for a tooth" is a typical example here. Nevertheless, a growing literature stream switched focus to another nonnegligible type of punishment, the one by third parties who are independent of or are only indirectly affected by those whom they punish $[25,26]$. Third-party punishment is a pure altruistic behavior. For instance, Fehr and Gachter study the enforcement mechanisms behind social norms, indicating that a large percentage of subjects are willing to enforce distribution and cooperation norms, although they incur costs and reap no economic benefits from their sanctions nor have they been directly harmed by the norm violation [25]. A stranger defending the weak against the strong is a good example. In short, third-party punishment is an important tool to maintain social norms in society.

In most studies, second- and third-party punishments are discussed together and are generally regarded as altruistic punishment types. However, despite both being altruistic behaviors, there are some differences between them. Some studies focus on the mechanisms of second- and thirdparty punishments and the impacts of anger on these punishment types show that the anger about personal harm, which is a trigger of second-party punishment, is a distinct emotion from the empathic anger due to witnessing injustice or harm to someone else, which is a trigger of third-party punishment $[25,27]$. That is, although both triggered emotions represent anger, they have different elicitors, thus causing different punishment. Bendor et al. identify that second-party punishment is easily affected by personal or situational factors; in other words, third-party punishment is more stable than second-party punishment [28]. Leibbrandt et al. prove that many third parties do not punish in an impartial or normative manner [26]. However, only few studies have investigated the effects of second- and thirdparty punishments on cooperation using experimental methods. For example, Zhou et al. find that third parties punish more frequently, severely, and less antisocially, resulting in a higher contribution level than that driven by second-party punishment [24].

While these studies inform the relationship between altruistic punishment and cooperative behaviors, there is currently no comparative study of the promotion effects of second- and third-party punishments on knowledge sharing that utilizes an evolutionary game model and an ensuing simulation. We thus analyze knowledge sharing behaviors by introducing an evolutionary game model with second-, third-, and mixed second-and-third-party punishment mechanisms to determine which one improves knowledge sharing best within an organization. From the ensuing Matlab programming simulation, we can better understand the effects of altruistic punishment on knowledge sharing and the differences between secondand third-party punishments.
2.3. Status Conflict and Knowledge Sharing. The employees in an organization have the need to pursue not only tangible resources, such as money, but also intangible resources, such as status [29]. However, status is pervasive in human behavior, both in the work environment and in everyday life [30], and the desire for status is a fundamental human motive. Status is defined as the respect and admiration, and voluntary deference individuals are afforded by others, thus being distinct from related constructs such as power, financial success, and social belongingness [31]. People's subjective well-being, self-esteem, and mental and physical health depend on the status level they are accorded by others, the importance of status being observed across individuals who differed in culture, gender, age, and personality, supporting the universality of the status motive [29, 31]. During the process of an individual's status pursuit, status conflicts arise when individuals attempts to protect or enhance their relative status.

As a sustainable competitive advantage, knowledge- especially professional knowledge-can enable employees to obtain status and power [32]. Prior research indicates that employees will use knowledge sharing as an interpersonal strategy to gain recognition and respect, thus improving their social prestige [33]. Additionally, individuals can show superiority compared to peers and expert authorities by sharing knowledge to boost their reputation and status [33-35]. The research on knowledge sharing from the perspective of status conflict motivation also proves that the individuals who are promotion-focused show a higher willingness to take risks, use this approach as a behavioral strategy, and are more likely to explore the advantages of novel behavior directions [36]. In short, they are more inclined to share knowledge with others. Nevertheless, knowledge sharing not only brings employees advantages in a status conflict but has also a disadvantage. Namely, without the control of knowledge, especially expertise, knowledge holders lose the competitive advantage from knowledge, which threatens directly their power and status in the organization [11,37]. Previous studies show that knowledge sharing may lead individuals to lose unique value and affects their competitive advantage, thus threatening their status $[13,38,39]$. Therefore, sharing knowledge brings both benefits and losses to individuals. Generally, knowledge owners gain respect and prestige by sharing knowledge, which has a positive impact on status conflicts; however, knowledge contributors also lose the control of their expertise, which has a negative effect on status conflicts. In this paper, we consider concurrently the positive and negative influences on status conflict and denote them as status conflict advantages and disadvantages, respectively.

2.4. Guanxi and Knowledge Sharing. Guanxi is a stable and enduring feature of China's national culture, as well as a source of security and protection in an unpredictable society [40]. Guanxi is today among the most important, talked about, and studied phenomena in China, lying at the heart of China's social order, its economic structure, and changing institutional landscape. It is considered important in almost 
every area of life from politics to business and from officialdom to street life and as informal connection, thus being also essential to gaining approval for or access to just about everything in China [41-43]. In other words, a key aspect of Chinese culture is being based on guanxi [44], which is different from the concepts of interpersonal relationships or social networks in western society. The Chinese phrase "guanxi" consists of two characters: "guan" means a gate or a hurdle and "xi" refers to a tie, a relationship, or a connection. Therefore, guanxi literally means "pass the gate and get connected" [41]. In summary, guanxi refers to interpersonal relationships or connections and can be applied not only to kinship and friendship relationships but also to social connections, such as dyadic relationships [45, 46].

Prior works on guanxi have contributed to the improved understanding of guanxi in China. For instance, Fei published his book "From the Soil: The Foundation of Chinese Society" [44] in 1940s after 10 years of field study, pointing out the differences between the U.S. society, which is organized by voluntary associations, and the Chinese society, which is organized by concentric guanxi circles. The Chinese are self-centered in communicating with others, extending from the family (the core) to relatives, friends, and so on. People who are farther away from the center of the circle are more alienated. The core Chinese values are the differentiated attitudes towards parents, children, siblings, kinsmen, friends, and so on, which Fei calls "differentiated modes of association" [44]. In the Chinese context, individual rights are not universalistic but guanxi-specific and particularistic_that is, no tie, no obligation, and no rights [41, 44]. Jacobs, a western scholar, was the first one to study relationships in the context of China in Taiwan. He uses the English transliteration "kuan-hsi" to express relationships and argues that the Chinese have long suggested that particularistic ties, or the so-called kuan-hsi, play an important role in politics [45]. Chiao [47] uses the Chinese Pinyin "guanxi" to refer to relationships in China, marking the uniqueness of relationships in the Chinese culture compared with western culture. One of the most representative studies on guanxi is Huang's "Human feelings and face: A game of Chinese power" [46], whose investigation of guanxi and "face" deepens the understanding of guanxi in China. Based on this prior study, Yang [48] categorizes guanxi in China into three groups: between family members; between familiar people, such as neighbors, friends, and colleagues; and between strangers or mere acquaintances. These three relationship categories have completely different social and psychological meanings to the involved parties and are governed by different sets of interpersonal rules.

A guanxi strategy can be defined as the development and use of personal networks by managers to gain status [40]. Knowledge sharing is a type of interpersonal interaction behavior among individuals. That is, individuals can obtain status under a guanxi strategy by means of sharing knowledge. Therefore, according to the theory of the "differentiated modes of association," knowledge owners make decisions on whether or how much to share their knowledge with others based on guanxi, defined as the location of a concentric circle from the center. Hence, we introduce the variable guanxi in our model to represent the Chinese cultural content.

2.5. Applying Evolutionary Game Theory in Analyzing Knowledge Sharing. The basic assumption in classical game theory is that agents are "completely rational," which leads to limitations in analyzing practical problems, because, in real life, agents are bonded rational instead of completely rational. Evolutionary game theory uses the basic assumption of individuals' bonded rationality and combines game theoretic analysis with dynamic evolution. It considers the equilibrium when individuals with bounded rationality seek optimization over time, namely, it depicts the evolution of a game over time. Therefore, evolutionary game theory is a useful tool for discussing the behaviors of individuals with bonded rationality and a refinement of the Nash equilibrium, thus extending and expanding the classic game theory.

While previous works regarding knowledge sharing have helped us better understand knowledge sharing behaviors, most of them are empirical. Therefore, these studies have several limitations, such as the use of variables based on a questionnaire completed by a single source over one time period [12]. As such, more research on knowledge sharing using different research methods is needed. Some scholars have investigated knowledge sharing from the perspective of social dilemmas in realistic organizations. The frameworks presented in these studies come from sociological research on cooperation and inform the sociopsychological processes governing exchanges among employees [2]. For example, Lin studies the relationship between knowledge sharing and indirect reciprocity with computational experiment [49]. These studies broaden the scope of the investigation on knowledge sharing, but further explorations are still required.

Nowadays, game theory has become one of the most common methods used to solve social dilemmas [50], along with evolutionary game theory, whose assumptions are based on learning ability and bounded rationality instead of complete rationality [51]. In evolutionary game theory, agents need to improve their strategies by imitating, copying, and learning constantly, after which the game eventually reaches stability, which is also known as the "evolutionary stable strategy" (ESS). Employees' knowledge sharing behaviors within an organization can be regarded as infinite dynamic repeated games, and their strategies may lead to conflicts of interests among relevant individuals. Meanwhile, free riding is a strategy that can bring individuals maximum benefits. Under these circumstances, evolutionary game theory can be used to explore knowledge sharing behaviors.

Few studies have hitherto discussed knowledge sharing between organizations by applying evolutionary game theory. For instance, Li et al. construct an evolutionary game model to analyze the dynamic evolution process of a firm's knowledge sharing behavior in a setting of supply chain networks [52], while Wang et al. investigate the behavioral characteristics of interfirm knowledge sharing in innovation clusters [53]. As there is currently no comparative study on the promotion effects of second- and third-party 
punishments on knowledge sharing that uses evolutionary game theory and an ensuing simulation, we aim to tackle this approach.

\section{Model}

3.1. Variables. To better describe the influence of knowledge on knowledge sharing, we classify knowledge into two types-general and professional knowledge-according to their complexity, flowability, and natural and social attributes. General knowledge refers to the open or universal knowledge in the organization, including rules and regulations, values, and culture, while professional knowledge refers to knowledge related to the product, technology, market, and operation of an organization. That is, professional knowledge rather refers to personal knowledge, experience, skills, or insight. These two types of knowledge are valued differently within organizations [54]. When individuals perceive the knowledge they possess as a valuable commodity, knowledge sharing becomes a process mediated by the decisions of what knowledge to share, when to share, and who to share it with [55]. Therefore, the type of knowledge affects the knowledge sharing behavior. General knowledge is easy to be shared, and employees can obtain it with a low cost; conversely, professional knowledge is difficult to share, since it is more valuable.

To simplify our model, we assume that the players in the evolutionary game are two employees from one organization. This game can be considered as an infinite dynamic repeated game, where each player has two options in selecting his/her strategy as either KS (knowledge sharing) or NKS (no knowledge sharing). It is impossible for either of these two players to fully understand the other one's utility function, which makes the situation an incomplete information game based on bounded rationality. Each player knows his/her own circumstances, including knowledge capacity, guanxi, status, and the gain and loss from sharing knowledge. Therefore, the decision on whether to share knowledge depends on the judgement of his/her expected revenue. Our model includes a number of variables, defined as follows.

3.1.1. $k_{1 P}, k_{1 G}, k_{2 P}, k_{2 G}$ : Knowledge Capacity. It denotes the maximal quantity of sharable knowledge for the two players. The knowledge is classified into two categories: professional and general knowledge. Hence, $k_{1 P}$ and $k_{1 G}$ refer to the maximal quantity of sharable professional and general knowledge, respectively, for the first player, and $k_{2 P}$ and $k_{2 G}$ refer to the maximal quantity of sharable professional and general knowledge, respectively, for the second player. Therefore, the sums of the sharable knowledge for these two players are $k_{1 P}+k_{1 G}$ and $k_{2 P}+k_{2 G}$, respectively.

3.1.2. $\alpha_{1}, \alpha_{2}$ : Guanxi Index. It refers to one player's perception of guanxi with the other player. Each player decides how to interact with the other one based on the theory of the "differentiated mode of association." That is, a closer guanxi leads to increases responsibility and less attention paid to payback, which translates as a higher willingness to share knowledge. Under the influence of guanxi, the actual sums of sharable knowledge for the two players are $\alpha_{1}\left(k_{1 P}+k_{1 G}\right)$ and $\alpha_{2}\left(k_{2 P}+k_{2 G}\right)$.

We concurrently consider the positive and negative influences of status conflict when players choose to share knowledge, which are described as status conflict advantage and disadvantage, respectively. The variables are as follows.

3.1.3. $\beta_{1 P}^{+}, \beta_{2 P}^{+}$: Status Conflict Advantage Coefficient from Sharing Professional Knowledge. This is the effect of status conflict advantage when a player shares professional knowledge. Individuals can boost their reputation by sharing professional knowledge, which has a positive effect on status conflict. The status conflict advantage of these two players from sharing professional knowledge is reflected in the status conflict advantage coefficient and the quantities of professional knowledge they actually share, respectively, denoted as $\beta_{1 P}^{+} \alpha_{1} k_{1 P}$ and $\beta_{2 P}^{+} \alpha_{2} k_{2 P}$.

\subsection{4. $\beta_{1 G}^{+}, \beta_{2 G}^{+}$: Status Conflict Advantage Coefficient from} Sharing General Knowledge. This is the effect of the status conflict advantage of a player when he/she shares general knowledge. As an interpersonal strategy, sharing general knowledge can also bring status conflict advantage to the individual. The status conflict advantages of the two players from sharing general knowledge are reflected in the status conflict advantage coefficient and the quantities of general knowledge they actually share, respectively, denoted as $\beta_{1 G}^{+} \alpha_{1} k_{1 G}$ and $\beta_{2 G}^{+} \alpha_{2} k_{2 G}$. Additionally, individuals can obtain a higher advantage from sharing professional knowledge compared to sharing general knowledge because professional knowledge is more valuable and they can better demonstrate their professional and technical skills. Therefore, we assume that $\beta_{1 P}^{+}>\beta_{1 G}^{+}, \beta_{2 P}^{+}>\beta_{2 G}^{+}$.

\subsection{5. $\beta_{1 P}^{-}, \beta_{2 P}^{-}$: Status Conflict Disadvantage Coefficient from} Sharing Professional Knowledge. This is the effect variable of the status conflict disadvantage of a player when he/she shares professional knowledge. Sharing general knowledge does not bring individuals a status conflict disadvantage because general knowledge is easy to share. Conversely, sharing professional knowledge means losing the control of expertise for an individual, which then threatens directly his/ her power and status. The status conflict disadvantage from sharing professional knowledge for these two players is reflected in the status conflict disadvantage coefficient and the quantities of professional knowledge they actually share, respectively, denoted as $\beta_{1 P}^{-} \alpha_{1} k_{1 P}$ and $\beta_{2 P}^{-} \alpha_{2} k_{2 P}$.

3.1.6. $\tau_{1 P}, \tau_{2 P}$ : Professional Knowledge Absorptive Capacity. This is the effect variable of the professional knowledge that a player can understand and absorb. In our model, we define the direct excess benefit as the benefit of the free rider from absorbing professional knowledge when he/she chooses NKS and the other player chooses KS. The direct excess benefit of the free rider is influenced by his/her professional 
knowledge absorptive capacity and the quantity of professional knowledge the other player actually shares and can be expressed as $\tau_{1 P} \alpha_{2} k_{2 P}$ and $\tau_{2 P} \alpha_{1} k_{1 P}$ for the two players, respectively.

3.1.7. $\tau_{1 G}, \tau_{2 G}$ : General Knowledge Absorptive Capacity. This is the effect variable of the general knowledge that a player can understand and absorb. In our model, we define the direct benefit as the benefit of the free rider from absorbing general knowledge when he/she chooses NKS and the other player chooses KS. The direct benefit of the free rider is related to his/her general knowledge absorption capacity and the quantity of general knowledge the other player actually shares and can be expressed as $\tau_{1 G} \alpha_{2} k_{2 G}$ and $\tau_{2 G} \alpha_{1} k_{1 G}$ for the two players, respectively. Additionally, professional knowledge is more difficult to be absorbed compared to general knowledge because of its characteristics. Hence, we assume that $\tau_{1 P}<\tau_{1 G}$, $\tau_{2 P}<\tau_{2 G}$.

3.1.8. $\Gamma_{1}, \Gamma_{2}$ : Synergy Revenue Coefficient of $K S$. It refers to the additional benefit generated from knowledge sharing. When both players share knowledge, they may create additional new knowledge due to knowledge fusion and, consequently, reciprocal benefits [52]. The synergy of the two players is affected by the sums of the professional and general knowledge they actually share, which can be expressed as $\Gamma_{1}\left[\alpha_{1}\left(k_{1 P}+k_{1 G}\right)+\alpha_{2}\left(k_{2 P}+k_{2 G}\right)\right]$ and $\Gamma_{2}\left[\alpha_{1}\left(k_{1 P}+k_{1 G}\right)+\alpha_{2}\left(k_{2 P}+k_{2 G}\right)\right]$, respectively.

$c_{1}, c_{2}$ : knowledge sharing cost. Based on transaction cost economics, KS incurs costs, including the opportunity, technical, and time costs that a sharer needs to pay.

$c_{s}$ : second-party punishment cost. Under second-party punishment, the punisher, as a player in the game, needs to pay the cost of punishing the free rider.

$f_{s}$ : penalty of the second-party punishment. Under second-party punishment, the free rider needs to pay the penalty of choosing NKS.

$f_{t}$ : penalty of the third-party punishment. Under thirdparty punishment, the free rider needs to pay for the penalty of choosing NKS.

The relevant parameters under the different punishment mechanisms are shown in Table 1.

3.2. Pay-Off Function Matrix. The pay-off functions for both players can be discussed under the following four scenarios.

(1) Both player1 and player2 choose KS. At this moment, the benefits and costs of both players include synergy revenue, status conflict advantage from sharing professional and general knowledge, status conflict disadvantage from sharing professional knowledge, and knowledge sharing cost. The pay-off functions of player1 and player2 are
$\Gamma_{1}\left[\alpha_{1}\left(k_{1 P}+k_{1 G}\right)+\alpha_{2}\left(k_{2 P}+k_{2 G}\right)\right]+\beta_{1 P}^{+} \alpha_{1} k_{1 P}+\beta_{1 G}^{+}$ $\alpha_{1} k_{1 G}-\beta_{1 P}^{-} \alpha_{1} k_{1 P}-c_{1}$ and $\Gamma_{2}\left[\alpha_{1}\left(k_{1 P}+k_{1 G}\right)+\alpha_{2}\right.$ $\left.\left(k_{2 P}+k_{2 G}\right)\right]+\beta_{2 P}^{+} \alpha_{2} k_{2 P}+\beta_{2 G}^{+} \alpha_{2} k_{2 G}-\beta_{2 P}^{-} \alpha_{2} k_{2 P}-c_{2}$, respectively.

(2) Player1 chooses KS and player2 chooses NKS. There can be three situations under this scenario, described as follows.

Situation 1: Second-party punishment is adopted. At this moment, player 2 is punished by player1. Player 1 needs to pay for the second-party punishment cost, $c_{s}$, while player2 needs to pay for the penalty of second-party punishment, $f_{s}$, since he/she chooses to be a free rider.

Situation 2: Third-party punishment is adopted. At this moment, player2 is punished by the independent third party. Player2 needs to pay the penalty of thirdparty punishment, $f_{t}$, since he/she chooses to be a free rider.

Situation 3: Mixed second-and-third-party punishment is adopted. At this moment, player2 is simultaneously punished by player1 and the independent third party. Player 1 needs to pay the second-party punishment cost, $c_{s}$, while player2 needs to pay the penalty of second-and-third-party punishment, $f_{s}+f_{t}$, since he/she chooses to be a free rider.

Based on these three situations, the benefits and costs of player 1 include the status conflict advantage from sharing professional and general knowledge, status conflict disadvantage from sharing professional knowledge, knowledge sharing cost, and secondparty punishment cost. The pay-off function of player1 is expressed as $\beta_{1 P}^{+} \alpha_{1} k_{1 P}+\beta_{1 G}^{+} \alpha_{1} k_{1 G}-$ $\beta_{1 P}^{-} \alpha_{1} k_{1 P}-c_{1}-c_{s}$. The benefits and costs of player2 include direct excess benefit, which is the benefit of the free rider from absorbing shared professional knowledge; direct benefit, which is the benefit of the free rider from absorbing shared general knowledge; and the penalty, which is from the second- or/and third-party punishment. The pay-off function of player2 is $\tau_{2 P} \alpha_{1} k_{1 P}+\tau_{2 G} \alpha_{1} k_{1 G}-f_{s}-f_{t}$.

(3) Player1 chooses NKS, and player2 chooses KS. Similar to scenario (2), the benefits and costs of player2 include the status conflict advantage from sharing professional and general knowledge, status conflict disadvantage from sharing professional knowledge, knowledge sharing cost, and secondparty punishment cost. The pay-off function of player2 is expressed as $\beta_{2 P}^{+} \alpha_{2} k_{2 P}+\beta_{2 G}^{+} \alpha_{2} k_{2 G}-\beta_{2 P}^{-}$ $\alpha_{2} k_{2 P}-c_{2}-c_{s}$. The benefits and costs of player 1 include direct excess benefit, direct benefit, and penalty, which are from the second- or/and thirdparty punishment. The pay-off function of player1 is $\tau_{1 P} \alpha_{2} k_{2 P}+\tau_{1 G} \alpha_{2} k_{2 G}-f_{s}-f_{t}$.

(4) Both player1 and player2 choose NKS. At this moment, both players have no benefits, since they do 
TABLE 1: Relevant parameters under different punishment mechanisms.

\begin{tabular}{lccc}
\hline \multirow{2}{*}{ Parameters } & \multicolumn{2}{c}{ Punishment mechanism } \\
& Second-party punishment & Third-party punishment & Mixed second-and-third-party punishments \\
\hline$c_{s}$ & $c_{s}>0$ & $c_{s}=0$ & $c_{s}>0$ \\
$f_{s}$ & $f_{s}>0$ & $f_{s}=0$ & $f_{s}>0$ \\
$f_{t}$ & $f_{t}=0$ & $f_{t}>0$ & $f_{t}>0$ \\
\hline
\end{tabular}

not share knowledge. Moreover, the second-party punishment is not implemented; thus, no one needs to pay for the penalty of second-party punishment. However, third-party punishment is present and both players need to pay for its penalty, $f_{t}$, since they both break the rule of cooperation. In other words, both players' pay-off is $-f_{t}$ under this scenario.

Overall, the pay-off function matrices under those four scenarios are summarized in Table 2.

\subsection{Evolutionary Stable Strategy}

3.3.1. Solving Evolutionary Stable Strategy by Applying Replicator Dynamic Equation. Based on the evolutionary game, we further assume that the initial probability of player1 choosing KS is $x$; thus, the initial probability of choosing NKS would be $(1-x)$. Similarly, the initial probability of player 2 choosing $\mathrm{KS}$ is $y$, meaning the initial probability of choosing NKS would be $(1-y)$. Moreover, $x$ and $y$ are functions of time.

The expected revenue of player 1 when he/she chooses KS is defined as per the following equation:

$$
\begin{aligned}
U_{1 Y}= & y\left\{\Gamma_{1}\left[\alpha_{1}\left(k_{1 P}+k_{1 G}\right)+\alpha_{2}\left(k_{2 P}+k_{2 G}\right)\right]+\beta_{1 P}^{+} \alpha_{1} k_{1 P}\right. \\
& \left.+\beta_{1 G}^{+} \alpha_{1} k_{1 G}-\beta_{1 P}^{-} \alpha_{1} k_{1 P}-c_{1}\right\}+(1-y)\left(\beta_{1 P}^{+} \alpha_{1} k_{1 P}\right. \\
& \left.+\beta_{1 G}^{+} \alpha_{1} k_{1 G}-\beta_{1 P}^{-} \alpha_{1} k_{1 P}-c_{1}-c_{s}\right) .
\end{aligned}
$$

The expected revenue of player1 when he/she chooses NKS is defined in the following equation:

$$
U_{1 N}=y\left(\tau_{1 P} \alpha_{2} k_{2 P}+\tau_{1 G} \alpha_{2} k_{2 G}-f_{s}-f_{t}\right)+(1-y)\left(-f_{t}\right) \text {. }
$$

The expected average revenue of player 1 is defined as per the following equation:

$$
\overline{U_{1}}=x U_{1 Y}+(1-x) U_{1 N} .
$$

Based on symmetry, the expected revenues of player2 when he/she chooses KS and NKS, and the expected average revenue are, respectively, expressed as per the following equations:

$$
\begin{aligned}
U_{2 Y}= & x\left\{\Gamma_{2}\left[\alpha_{1}\left(k_{1 P}+k_{1 G}\right)+\alpha_{2}\left(k_{2 P}+k_{2 G}\right)\right]\right. \\
& \left.+\beta_{2 P}^{+} \alpha_{2} k_{2 P}+\beta_{2 G}^{+} \alpha_{2} k_{2 G}-\beta_{2 P}^{-} \alpha_{2} k_{2 P}-c_{2}\right\} \\
& +(1-x)\left(\beta_{2 P}^{+} \alpha_{2} k_{2 P}+\beta_{2 G}^{+} \alpha_{2} k_{2 G}-\beta_{2 P}^{-} \alpha_{2} k_{2 P}-c_{2}-c_{s}\right),
\end{aligned}
$$

$$
U_{2 N}=x\left(\tau_{2 P} \alpha_{1} k_{1 P}+\tau_{2 G} \alpha_{1} k_{1 G}-f_{s}-f_{t}\right)+(1-x)\left(-f_{t}\right) \text {, }
$$

$$
\overline{U_{2}}=y U_{2 Y}+(1-y) U_{2 N} .
$$

Then, we apply the replicator dynamic equation for player1 and player2 as

$$
\begin{aligned}
F(x)= & \frac{\mathrm{d} x}{\mathrm{~d} t}=x\left(U_{1 Y}-\overline{U_{1}}\right)=x(1-x)\left(U_{1 Y}-U_{1 N}\right) \\
= & x(1-x)\left\{y \Gamma_{1}\left[\alpha_{1}\left(k_{1 P}+k_{1 G}\right)+\alpha_{2}\left(k_{2 P}+k_{2 G}\right)\right]\right. \\
& -y \tau_{1 P} \alpha_{2} k_{2 P}-y \tau_{1 G} \alpha_{2} k_{2 G}+y c_{s}+y f_{s}+\beta_{1 P}^{+} \alpha_{1} k_{1 P} \\
& \left.+\beta_{1 G}^{+} \alpha_{1} k_{1 G}-\beta_{1 P}^{-} \alpha_{1} k_{1 P}-c_{1}-c_{s}+f_{t}\right\} \\
F(y)= & \frac{\mathrm{d} y}{\mathrm{~d} t}=y\left(U_{2 Y}-\overline{U_{2}}\right)=y(1-y)\left(U_{2 Y}-U_{2 N}\right) \\
= & y(1-y)\left\{x \Gamma_{2}\left[\alpha_{1}\left(k_{1 P}+k_{1 G}\right)+\alpha_{2}\left(k_{2 P}+k_{2 G}\right)\right]\right. \\
& -x \tau_{2 P} \alpha_{1} k_{1 P}-x \tau_{2 G} \alpha_{1} k_{1 G}+x c_{s}+x f_{s}+\beta_{2 P}^{+} \alpha_{2} k_{2 P} \\
& \left.+\beta_{2 G}^{+} \alpha_{2} k_{2 G}-\beta_{2 P}^{-} \alpha_{2} k_{2 P}-c_{2}-c_{s}+f_{t}\right\}
\end{aligned}
$$

To solve the stable strategy in equations (7) and (8), we need to obtain the critical value.

If $F(x)=0$, we get $x^{*}=0$, or $x^{*}=1$, or

$$
y^{*}=\frac{\left(\beta_{1 P}^{-} \alpha_{1} k_{1 P}+c_{1}+c_{s}-\beta_{1 P}^{+} \alpha_{1} k_{1 P}-\beta_{1 G}^{+} \alpha_{1} k_{1 G}-f_{t}\right)}{\left\{\Gamma_{1}\left[\alpha_{1}\left(k_{1 P}+k_{1 G}\right)+\alpha_{2}\left(k_{2 P}+k_{2 G}\right)\right]-\tau_{1 P} \alpha_{2} k_{2 P}-\tau_{1 G} \alpha_{2} k_{2 G}+c_{s}+f_{s}\right\}},
$$


TABle 2: Pay-off function matrix.

Player 2

KS

NKS

KS

Player 1
$\Gamma_{1}\left[\alpha_{1}\left(k_{1 P}+k_{1 G}\right)+\alpha_{2}\left(k_{2 P}+k_{2 G}\right)\right]+\beta_{1 P}^{+} \alpha_{1} k_{1 P}+\beta_{1 G}^{+} \alpha_{1} k_{1 G}-\beta_{1 P}^{-} \alpha_{1} k_{1 P}-c_{1}$ $\Gamma_{2}\left[\alpha_{1}\left(k_{1 P}+k_{1 G}\right)+\alpha_{2}\left(k_{2 P}+k_{2 G}\right)\right]+\beta_{2 P}^{+} \alpha_{2} k_{2 P}+\beta_{2 G}^{+} \alpha_{2} k_{2 G}-\beta_{2 P}^{-} \alpha_{2} k_{2 P}-c_{2}$ $\tau_{1 P} \alpha_{2} k_{2 P}+\tau_{1 G} \alpha_{2} k_{2 G}-f_{s}-f_{t}$ $\beta_{2 P}^{+} \alpha_{2} k_{2 P}+\beta_{2 G}^{+} \alpha_{2} k_{2 G}-\beta_{2 P}^{-} \alpha_{2} k_{2 P}-c_{2}-c_{s}$ $\beta_{1 P}^{+} \alpha_{1} k_{1 p}+\beta_{1 G}^{+} \alpha_{1} k_{1 G}-\beta_{1 P}^{-} \alpha_{1} k_{1 p}-c_{1} c_{s}$ $\tau_{2 P} \alpha_{1} k_{1 P}+\tau_{2 G} \alpha_{1} k_{1 G}-f_{s}-f_{t}$

$-f_{t}$

If $F(y)=0$, we get $y^{*}=0$, or $y^{*}=1$, or

$$
x^{*}=\frac{\left(\beta_{2 P}^{-} \alpha_{2} k_{2 P}+c_{2}+c_{s}-\beta_{2 P}^{+} \alpha_{2} k_{2 P}-\beta_{2 G}^{+} \alpha_{2} k_{2 G}-f_{t}\right)}{\left\{\Gamma_{2}\left[\alpha_{1}\left(k_{1 P}+k_{1 G}\right)+\alpha_{2}\left(k_{2 P}+k_{2 G}\right)\right]-\tau_{2 P} \alpha_{1} k_{1 P}-\tau_{2 G} \alpha_{1} k_{1 G}+c_{s}+f_{s}\right\}},
$$

Then, it can be concluded that there are five evolutionary equilibrium points during the game: $(0,0),(0,1),(1,0),(1$, $1),\left(x^{*}, y^{*}\right)$.

Subsequently, the characteristics of the evolutionary equilibrium points can be analyzed based on the Jacobian matrix of the dynamic system [56]. According to equations (7) and (8), the Jacobian matrix can be expressed as

$$
\begin{aligned}
J= & \left(\begin{array}{cc}
a & b \\
c & d
\end{array}\right) \\
a= & (1-2 x)\left\{y \Gamma_{1}\left[\alpha_{1}\left(k_{1 P}+k_{1 G}\right)+\alpha_{2}\left(k_{2 P}+k_{2 G}\right)\right]\right. \\
& -y \tau_{1 P} \alpha_{2} k_{2 P}-y \tau_{1 G} \alpha_{2} k_{2 G}+y c_{s}+y f_{s}+\beta_{1 P}^{+} \alpha_{1} k_{1 P} \\
& \left.+\beta_{1 G}^{+} \alpha_{1} k_{1 G}-\beta_{1 P}^{-} \alpha_{1} k_{1 P}-c_{1}-c_{s}+f_{t}\right\} \\
b= & x(1-x)\left\{\Gamma_{1}\left[\alpha_{1}\left(k_{1 P}+k_{1 G}\right)+\alpha_{2}\left(k_{2 P}+k_{2 G}\right)\right]\right. \\
& \left.-\tau_{1 P} \alpha_{2} k_{2 P}-\tau_{1 G} \alpha_{2} k_{2 G}+c_{s}+f_{s}\right\} \\
c= & y(1-y)\left\{\Gamma_{2}\left[\alpha_{1}\left(k_{1 P}+k_{1 G}\right)+\alpha_{2}\left(k_{2 P}+k_{2 G}\right)\right]\right. \\
& \left.-\tau_{2 P} \alpha_{1} k_{1 P}-\tau_{2 G} \alpha_{1} k_{1 G}+c_{s}+f_{s}\right\} \\
d= & (1-2 y)\left\{x \Gamma_{2}\left[\alpha_{1}\left(k_{1 P}+k_{1 G}\right)+\alpha_{2}\left(k_{2 P}+k_{2 G}\right)\right]\right. \\
& -x \tau_{2 P} \alpha_{1} k_{1 P}-x \tau_{2 G} \alpha_{1} k_{1 G}+x c_{s}+x f_{s}+\beta_{2 P}^{+} \alpha_{2} k_{2 P} \\
& \left.+\beta_{2 G}^{+} \alpha_{2} k_{2 G}-\beta_{2 P}^{-} \alpha_{2} k_{2 P}-c_{2}-c_{s}+f_{t}\right\} .
\end{aligned}
$$

The value of the Jacobian matrix is

$$
\begin{aligned}
\operatorname{det}(\mathrm{J})= & a d-b c=(1-2 x)\left\{y \Gamma _ { 1 } \left[\alpha_{1}\left(k_{1 P}+k_{1 G}\right)\right.\right. \\
& \left.+\alpha_{2}\left(k_{2 P}+k_{2 G}\right)\right]-y \tau_{1 P} \alpha_{2} k_{2 P}-y \tau_{1 G} \alpha_{2} k_{2 G}+y c_{s} \\
& +y f_{s}+\beta_{1 P}^{+} \alpha_{1} k_{1 P}+\beta_{1 G}^{+} \alpha_{1} k_{1 G}-\beta_{1 P}^{-} \alpha_{1} k_{1 P} \\
& \left.-c_{1}-c_{s}+f_{t}\right\} \cdot(1-2 y) \\
& \cdot\left\{x \Gamma_{2}\left[\alpha_{1}\left(k_{1 P}+k_{1 G}\right)+\alpha_{2}\left(k_{2 P}+k_{2 G}\right)\right]-x \tau_{2 P} \alpha_{1} k_{1 P}\right. \\
& -x \tau_{2 G} \alpha_{1} k_{1 G}+x c_{s}+x f_{s}+\beta_{2 P}^{+} \alpha_{2} k_{2 P}+\beta_{2 G}^{+} \alpha_{2} k_{2 G} \\
& \left.-\beta_{2 P}^{-} \alpha_{2} k_{2 P}-c_{2}-c_{s}+f_{t}\right\}-x(1-x) \\
& \cdot\left\{\Gamma_{1}\left[\alpha_{1}\left(k_{1 P}+k_{1 G}\right)+\alpha_{2}\left(k_{2 P}+k_{2 G}\right)\right]-\tau_{1 P} \alpha_{2} k_{2 P}\right. \\
& \left.-\tau_{1 G} \alpha_{2} k_{2 G}+c_{s}+f_{s}\right\} \cdot y(1-y)\left\{\Gamma _ { 2 } \left[\alpha_{1}\left(k_{1 P}+k_{1 G}\right)\right.\right. \\
& \left.\left.+\alpha_{2}\left(k_{2 P}+k_{2 G}\right)\right]-\tau_{2 P} \alpha_{1} k_{1 P}-\tau_{2 G} \alpha_{1} k_{1 G}+c_{s}+f_{s}\right\} .
\end{aligned}
$$

The trace of the Jacobian matrix can be expressed as

$$
\begin{aligned}
\operatorname{tr}(J)= & a+d=(1-2 x)\left\{y \Gamma _ { 1 } \left[\alpha_{1}\left(k_{1 P}+k_{1 G}\right)\right.\right. \\
& \left.+\alpha_{2}\left(k_{2 P}+k_{2 G}\right)\right]-y \tau_{1 P} \alpha_{2} k_{2 P}-y \tau_{1 G} \alpha_{2} k_{2 G}+y c_{s} \\
& +y f_{s}+\beta_{1 P}^{+} \alpha_{1} k_{1 P}+\beta_{1 G}^{+} \alpha_{1} k_{1 G}-\beta_{1 P}^{-} \alpha_{1} k_{1 P} \\
& \left.-c_{1}-c_{s}+f_{t}\right\}+(1-2 y) \\
& +\left\{x \Gamma_{2}\left[\alpha_{1}\left(k_{1 P}+k_{1 G}\right)+\alpha_{2}\left(k_{2 P}+k_{2 G}\right)\right]\right. \\
& -x \tau_{2 P} \alpha_{1} k_{1 P}-x \tau_{2 G} \alpha_{1} k_{1 G}+x c_{s}+x f_{s}+\beta_{2 P}^{+} \alpha_{2} k_{2 P} \\
& \left.+\beta_{2 G}^{+} \alpha_{2} k_{2 G}-\beta_{2 P}^{-} \alpha_{2} k_{2 P}-c_{2}-c_{s}+f_{t}\right\} .
\end{aligned}
$$


Next, the value and trace of the Jacobian matrix at each evolutionary equilibrium point are calculated and the results shown in Table 3.

Afterwards, the evolutionary equilibrium points can be discussed, we define polynomials (1)-(6) as follows.

$$
\begin{aligned}
& \text { Polynomial } \\
& \Gamma_{1}\left[\alpha_{1}\left(k_{1 P}+k_{1 G}\right)+\alpha_{2}\left(k_{2 P}+k_{2 G}\right)\right]-\tau_{1 P} \alpha_{2} k_{2 P}- \\
& \tau_{1 G} \alpha_{2} k_{2 G} \\
& \text { Polynomial } \\
& \Gamma_{2}\left[\alpha_{1}\left(k_{1 P}+k_{1 G}\right)+\alpha_{2}\left(k_{2 P}+k_{2 G}\right)\right]-\tau_{2 P} \alpha_{1} k_{1 P}- \\
& \tau_{2 G} \alpha_{1} k_{1 G} \\
& \text { Polynomial } \\
& \beta_{1 P}^{+} \alpha_{1} k_{1 P}+\beta_{1 G}^{+} \alpha_{1} k_{1 G}-\beta_{1 P}^{-} \alpha_{1} k_{1 P}-c_{1}-c_{s}+f_{t} \\
& \text { Polynomial } \\
& \beta_{2 P}^{+} \alpha_{2} k_{2 P}+\beta_{2 G}^{+} \alpha_{2} k_{2 G}-\beta_{2 P}^{-} \alpha_{2} k_{2 P}-c_{2}-c_{s}+f_{t} \\
& \text { Polynomial } \\
& \Gamma_{1}\left[\alpha_{1}\left(k_{1 P}+k_{1 G}\right)+\alpha_{2}\left(k_{2 P}+k_{2 G}\right)\right]-\tau_{1 P} \alpha_{2} k_{2 P}- \\
& \tau_{1 G} \alpha_{2} k_{2 G}+\beta_{1 P}^{+} \alpha_{1} k_{1 P}+\beta_{1 G}^{+} \alpha_{1} k_{1 G}-\beta_{1 P}^{-} \alpha_{1} k_{1 P}-c_{1}+ \\
& f_{s}+f_{t} \\
& \text { Polynomial } \\
& \Gamma_{2}\left[\alpha_{1}\left(k_{1 P}+k_{1 G}\right)+\alpha_{2}\left(k_{2 P}+k_{2 G}\right)\right]-\tau_{2 P} \alpha_{1} k_{1 P}- \\
& \tau_{2 G} \alpha_{1} k_{1 G}+\beta_{2 P}^{+} \alpha_{2} k_{2 P}+\beta_{2 G}^{+} \alpha_{2} k_{2 G}-\beta_{2 P}^{-} \alpha_{2} k_{2 P}-c_{2}+ \\
& f_{s}+f_{t}
\end{aligned}
$$

Polynomial (1) refers to the difference between the synergy revenue and the sum of the direct excess benefit and direct benefit of player1. Polynomial (2) is the difference between the synergy revenue and the sum of the direct excess benefit and direct benefit of player2. The synergy revenue is the additional benefit generated from knowledge sharing when both players choose to share. The direct excess benefit is the revenue of the free rider from absorbing the professional knowledge that the other player shares and the direct benefit is the revenue of the free rider from absorbing the general knowledge that the other player shares. When both players choose KS, due to knowledge collision and fusion, the synergy revenue is greater than the sum of the direct excess benefit and direct benefit. Therefore, polynomials (1) and (2) are both greater than 0 , that is, (1) $>0$ and (2) $>0$.

When player2 chooses NKS, polynomial (3) indicates the difference between the benefit of player1 when he/she chooses KS and that when he/she chooses NKS. Similarly, when player1 chooses NKS, polynomial (4) expresses the difference between the benefit of player2 when he/she chooses KS and that when he/she chooses NKS.

When player2 chooses KS, polynomial (5) denotes the difference between the benefit of player1 when he/she chooses KS and that when he/she chooses NKS. Similarly, when player1 chooses KS, polynomial (6) represents the difference between the benefit of player2 when he/she chooses KS and that when he/she chooses NKS.

Additionally, according to the equation estimation, it can be concluded that (5) $=(1)+(3)+c_{s}+f_{s}$; (6) $=$ (2) + (4) $+c_{s}+f_{s}$.

Next, the differences of polynomials (3)-(6) are discussed (1) $>0$, and (2) $>0$ ).
On one hand, when (5) $<0$ ((5) = (1) + (3) $+c_{s}+f_{s}$ ), because (1) $>0$, it can be concluded that (3) $<0$; similarly, when (6) $<0$, it can be concluded that (4) $<0$. On the other hand, when (5) $>0$, we can draw the conclusion that (3) $>0$ and (3) $<0$ are both possible; similarly, when (6) $>0$, (4) $>0$ and (4) $<0$ are both possible. Therefore, there are nine stable states (situations I-IX) under which polynomials (3)-(6) satisfy different conditions, as shown in Table 4.

When the evolution stable states of the system are in situations I-III, origin $\mathrm{O}(0,0)$ is the evolutionary equilibrium point, and the evolutionary game phases are shown in Figure 1. When the evolution stable states of the system are in situations IV-VI, point $\mathrm{C}(1,1)$ is the evolutionary equilibrium point, and the evolutionary game phases are shown in Figure 2. When the evolution stable states of the system are in situations VII and VIII, points B $(1,0)$ and A $(0,1)$ are the evolutionary equilibrium points, respectively, and the evolutionary game phases are shown in Figure 3. Specifically, only in situation IX, that is, when (5) $>0$, (3) $<0$, and (6) $>0$, (4) $<0$, the system is able to satisfy $1>x^{*}>0$ and $1>y^{*}>0$ (for details, see Appendix A). At this moment, origin $\mathrm{O}(0,0)$ and point $\mathrm{C}(1,1)$ are the ESS and point $\mathrm{D}$ $\left(x^{*}, y^{*}\right)$ is saddle point. The evolutionary game phase diagram is shown in Figure 4.

Next, the evolutionary stable strategy of the system will be discussed further.

3.3.2. Analysis of the Evolutionary Stable Strategy. First, we discuss situations I-III. In situation I, that is, when (5) $<0$, (3) $<0$, and (6) $>0$, (4) $<0$, regardless what strategy player2 chooses, the benefit of player 1 from choosing NKS is greater than that from choosing $\mathrm{KS}$; therefore, player1 always chooses NKS. Player2 would eventually choose NKS, since player1 chooses NKS. In short, both players would ultimately choose NKS. Therefore, the ESS of the system is $\{\mathrm{NKS}, \mathrm{NKS}\}$. Similarly, in situation II, that is, when (5) $>0$, (3) $<0$, and (6) $<0$, (4) $<0$, player2 always chooses NKS and player1 would finally choose NKS based on player2's choice. Therefore, the ESS of the system is $\{\mathrm{NKS}, \mathrm{NKS}\}$. In situation III, that is, when (5) $<0$, (3) $<0$, and (6) $<0$, (4) $<0$, it can be concluded that, regardless what strategy a player chooses, the other player can obtain more benefits by choosing NKS than KS. Therefore, regardless of the initial KS probability in the game, both players would eventually choose NKS; therefore, the ESS of the system is $\{\mathrm{NKS}, \mathrm{NKS}\}$. The evolutionary game phases for situations I-III are shown in Figure 1.

Second, we discuss situations IV-VI. In situation IV, that is, when (5) $>0$, (3) $>0$, and (6) $>0$, (4) $<0$, regardless what strategy player2 chooses, the benefit of player 1 from choosing $\mathrm{KS}$ is greater than that from choosing NKS; therefore, player1 always chooses KS. Player2 would finally choose KS, since player1 chooses KS. In short, both players would eventually choose KS. Therefore, the ESS of the system is $\{\mathrm{KS}, \mathrm{KS}\}$. Similarly, in situation V, that is, when (5) $>0$, (3) $<0$, and (6) $>0$, (4) $>0$, player2 always chooses $\mathrm{KS}$, and player1 would finally choose KS based on player2's choice. Therefore, the ESS of the system is $\{\mathrm{KS}, \mathrm{KS}\}$. In situation VI, that is, when (5) $>0$, (3) $>0$, and (6) $>0$, (4) $>0$, it 


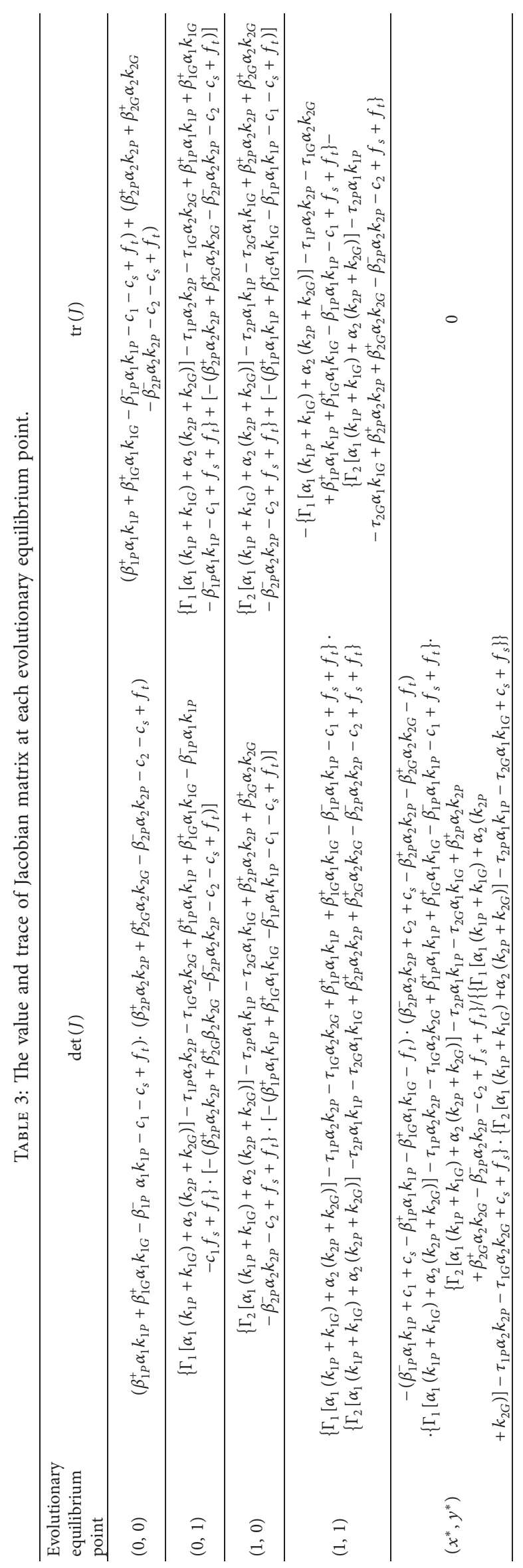




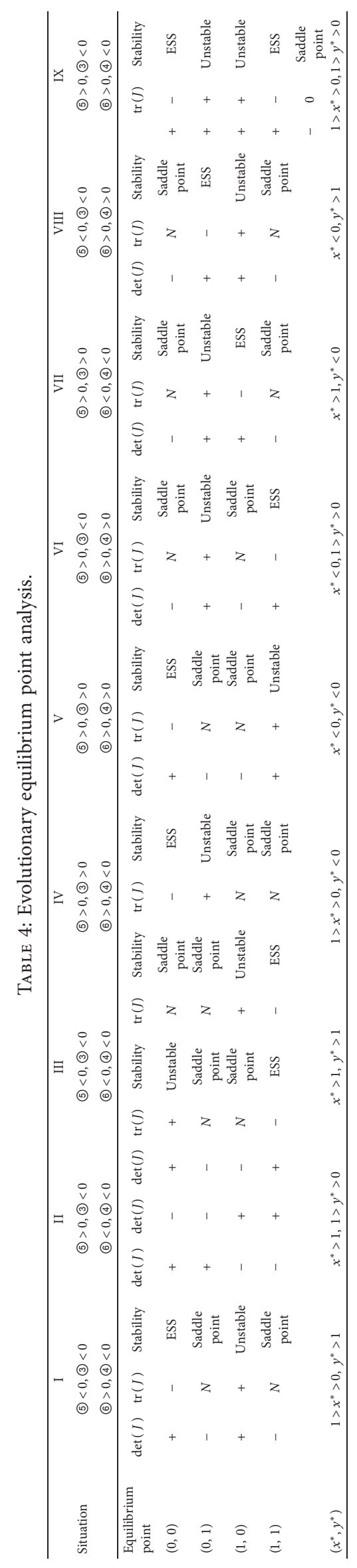




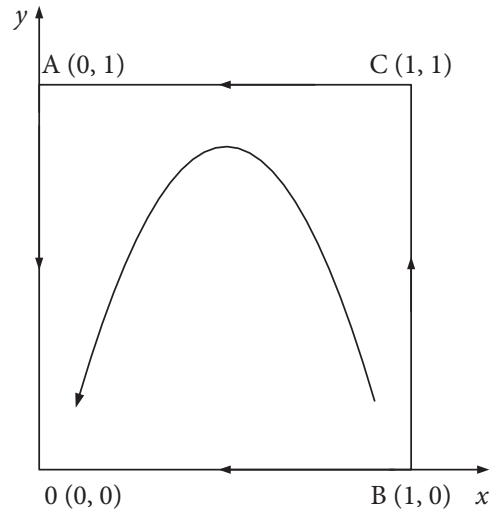

(a)

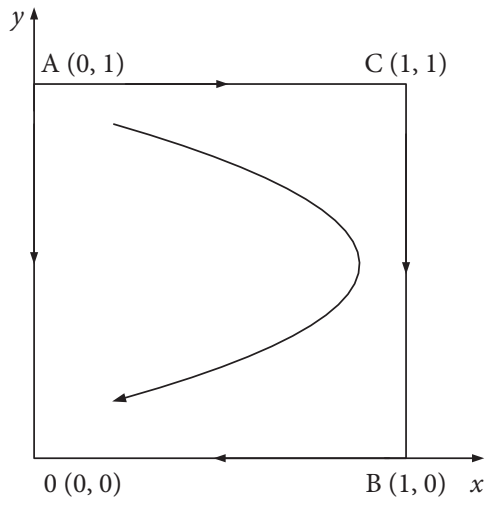

(b)

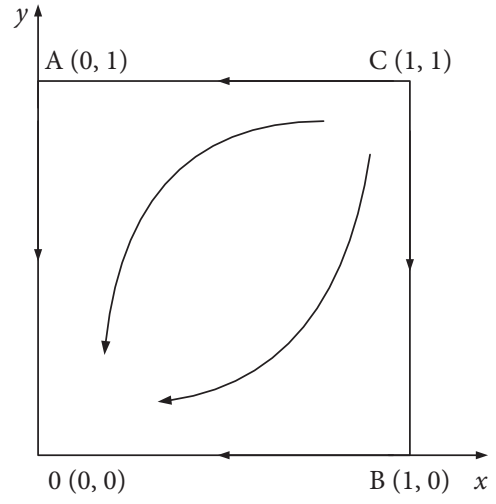

(c)

FIgURE 1: Evolutionary game phase diagrams (when origin O $(0,0)$ is the ESS). (a) Situation I. (b) Situation II. (c) Situation III.

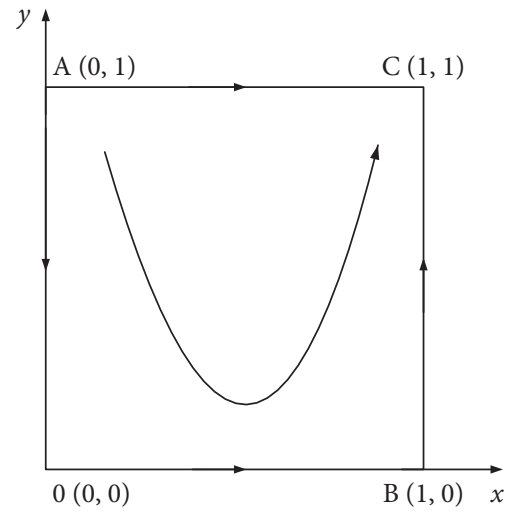

(a)

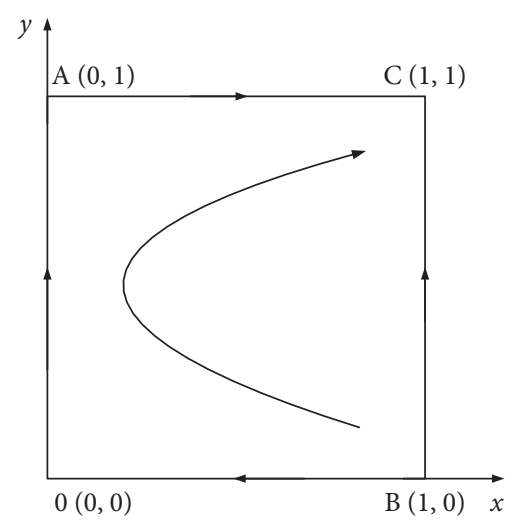

(b)

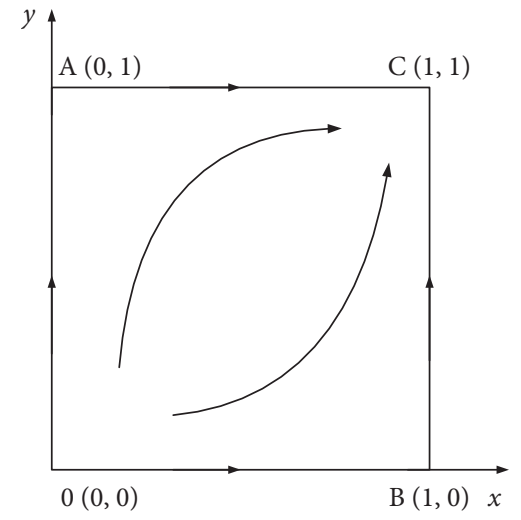

(c)

FIgURE 2: Evolutionary game phase diagrams (when point C $(1,1)$ is the ESS). (a) Situation IV. (b) Situation V. (c) Situation VI.

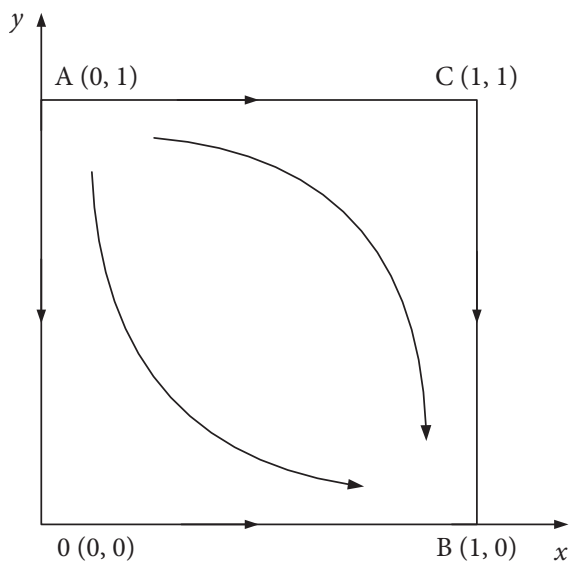

(a)

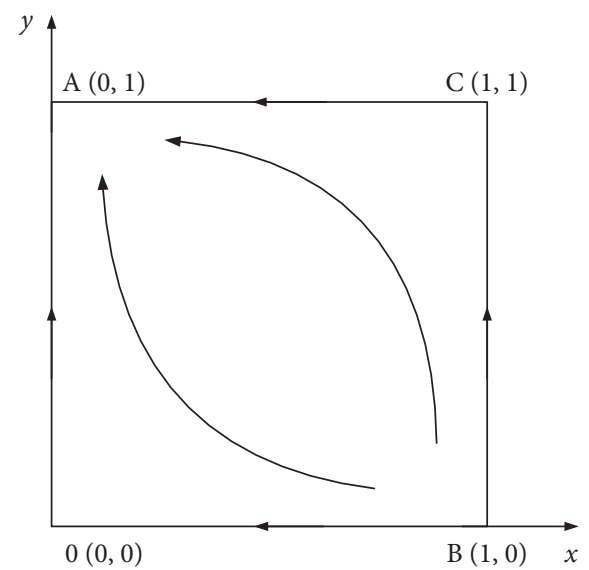

(b)

FIGURE 3: Evolutionary game phases diagrams. In situation VII, the ESS is point B $(1,0)$. In situation VIII, the ESS is point A $(0,1)$. (a) Situation VII. (b) Situation VIII. 


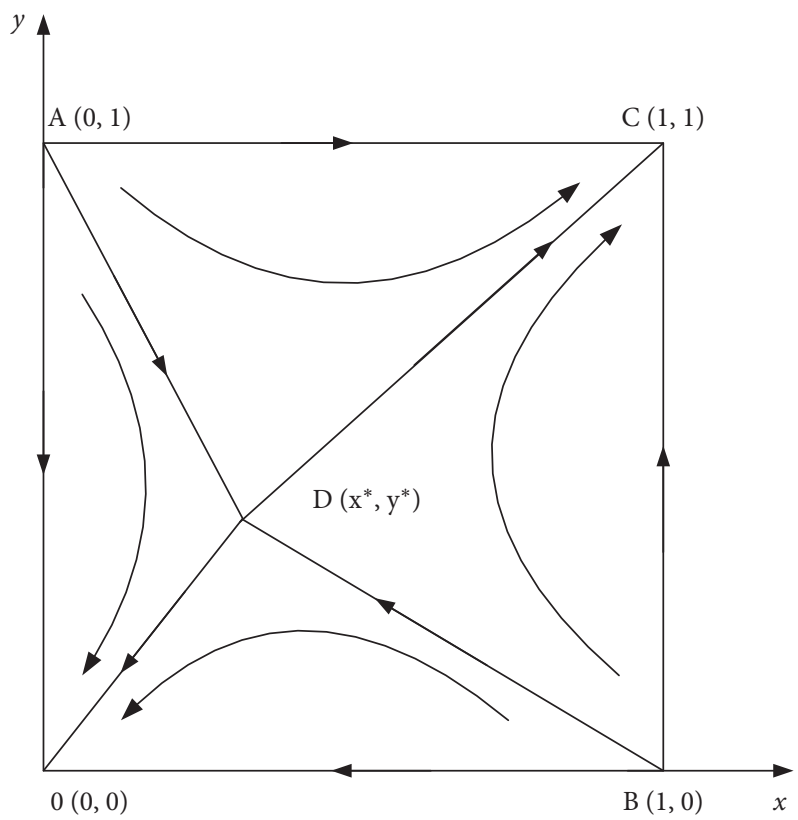

FIGURE 4: Evolutionary game phases diagram in situation IX. In this situation, origin $\mathrm{O}(0,0)$ and point $\mathrm{C}(1,1)$ are $\mathrm{ESS}$, and point $\mathrm{D}\left(x^{*}, y^{*}\right)$ is the saddle point.

can be concluded that, regardless what strategy one player chooses, the other can obtain more benefits from choosing KS than NKS. Therefore, regardless of the initial KS probability, both players would ultimately choose KS; therefore, the ESS of the system is $\{\mathrm{KS}, \mathrm{KS}\}$. The evolutionary game phases for situations IV-VI are shown in Figure 2.

Third, we discuss situations VII and VIII. In situation VII, that is, when (5) $>0$, (3) $>0$, and (6) $<0$, (4) $<0$, regardless what strategy player 2 chooses, the benefit of player 1 from choosing $\mathrm{KS}$ is greater than that from choosing NKS; therefore, player1 always chooses KS. However, for player2, regardless what strategy player1 chooses, the benefit of player 2 from choosing NKS is greater than that from choosing KS; thus, player2 always chooses NKS. In short, the ESS of the system is $\{\mathrm{KS}, \mathrm{NKS}\}$. Similarly, in situation VIII, that is, when (5) $<0$, (3) $<0$, and (6) $>0$, (4) $>0$, player1 always chooses NKS and player 2 always chooses KS. In sum, the ESS of the system is $\{\mathrm{NKS}, \mathrm{KS}\}$. The evolutionary game phases for situations VII and VIII are shown in Figure 3.

Finally, we discuss situation IX, that is, when (5) $>0$, (3) $<0$, and (6) $>0$, (4) $<0$. From Table 4, there are five equilibrium points of the system, namely, $(0,0),(0,1),(1,0)$, $(1,1)$, and $\left(x^{*}, y^{*}\right)$. Among them, origin $\mathrm{O}(0,0)$ and point $\mathrm{C}$ $(1,1)$ are ESS, that is, $\{$ NKS, NKS $\}$ and $\{$ KS, KS $\}$ could both be the evolutionary stable strategy set of the system. In other words, these two players either both choose NKS or KS in the evolutionary stable state. The evolutionary game phases diagram for situation IX is shown in Figure 4.

Additionally, point $\mathrm{D}\left(x^{*}, y^{*}\right)$ is the saddle point. Polyline ADB, formed by points A $(0,1), \mathrm{D}\left(x^{*}, y^{*}\right)$, and $\mathrm{B}$ $(1,0)$, is a critical line for the system to converge to different evolutionary stable states. In area OADB, by continuously learning and improving, both players find out that NKS is better-off than KS, and the system converges to origin $\mathrm{O}(0,0)$, which means strategy set $\{\mathrm{NKS}, \mathrm{NKS}\}$ is the stable result and the behavior of sharing knowledge disappears. However, in area CADB, the system converges to point $C(1,1)$, which shows that strategy set $\{\mathrm{KS}, \mathrm{KS}\}$ is a stable result and the behavior of free-riding disappears. In this situation, the stable result of the system is not only related to the initial probabilities of the knowledge sharing of the two players but also to the value of saddle point $\mathrm{D}\left(x^{*}, y^{*}\right)$. Because area OADB can be expressed as $S_{\mathrm{OADB}}=1 / 2\left(x^{*}+y^{*}\right)$, when $x^{*}$ and $y^{*}$ decrease, area OADB would also be reduced. In the meantime, area CADB is increasing; thus, the probability of $\mathrm{KS}$ increases and vice versa. Therefore, the value of saddle point $\mathrm{D}\left(x^{*}, y^{*}\right)$ is inversely proportional to area $\mathrm{CADB}$ and the probabilities of KS for both players.

3.3.3. Further Discussion of Situation IX with Universal Dilemma Strength. The concept of "dilemma strength" was first put forward by Tanimoto and Sagara [57], who suggest that pairwise games can be classified based on gamble-intending (GID) and risk-averting (RAD) dilemmas, respectively, defined by $D_{g}=T-R$ and $D_{r}=P-S$. $R$ denotes a reward for mutual cooperation (C) and $P$ is the punishment for mutual defection (D). However, when one chooses $\mathrm{C}$ and the other $\mathrm{D}$, the latter gets temptation pay-off, $\mathrm{T}$, while the former is left with the sucker's pay-off, S. Furthermore, they point out that, when $D_{g}>0$ and $D_{r}>0$, the game becomes the prisoner's dilemma (PD), whereby $\mathrm{D}$ dominates C. When $D_{g}<0$ and $D_{r}<0$, we have the harmony $(\mathrm{H})$ game, whereby $\mathrm{C}$ dominates D (i.e., no dilemma exists). When $D_{g}>0$, and $D_{r}<0$, we deal with the chicken $(\mathrm{CH}$, also known as snowdrift (SD) or hawk-dove (HD)) game, which has an 
internal (polymorphic) equilibrium. Finally, when $D_{g}<0$ and $D_{r}>0$, the game is the stag hunt $(\mathrm{SH})$, which is characterized by bistability. Therefore, $D_{g}$ and $D_{r}$ are useful in quantifying the dilemma strength and determining the replicator dynamic and its internal equilibrium. However, Wang et al. [58] derive that parameters $D_{g}$ and $D_{r}$ are insufficient to accurately predict the evolution of cooperation in a pairwise game when a reciprocity mechanism is introduced into it. Therefore, they modify the parameters for the dilemma strength, which is useful for the universal comparison of the various reciprocity mechanisms supported by different models. They introduce a new set of scaling parameters by defining new GID and RAD as $D_{g}^{\prime}$ and $D_{r}^{\prime}$, respectively:

$$
D_{g}^{\prime}=\frac{T-R}{R-P}=\frac{D_{g}}{R-P}, D_{r}^{\prime}=\frac{P-S}{R-P}=\frac{D_{r}}{R-P} .
$$

Further study [59] discusses the universal dilemma strength and its applications, and the results demonstrate that the new scaling for dilemma strength is sufficient to estimate the dilemma possibility of the game structure and can cover the range from an infinite and well-mixed population to a finite and spatially-structured one.

Our model is structured with the characteristics of the infinite and well-mixed populations with social viscosity; therefore, in the following section, we further discuss situation IX (as shown in Table 4 and Figure 4) in terms of the universal scaling for dilemma strength.

(1) Game Structure and Universal Dilemma Strength. Considering the heterogeneity of the two players, we present the pay-off function matrices for player1 and player2 as equations (15) and (16), respectively:

\section{KS NKS}

$$
\begin{aligned}
A= & \mathrm{KS}\left(\begin{array}{cc}
R & S \\
T & P
\end{array}\right), \\
& \mathrm{NKS}=\Gamma_{1}\left[\alpha_{1}\left(k_{1 P}+k_{1 G}\right)+\alpha_{2}\left(k_{2 P}+k_{2 G}\right)\right] \\
& +\beta_{1 P}^{+} \alpha_{1} k_{1 P}+\beta_{1 G}^{+} \alpha_{1} k_{1 G}-\beta_{1 P}^{-} \alpha_{1} k_{1 P}-c_{1}, \\
S= & \beta_{1 P}^{+} \alpha_{1} k_{1 P}+\beta_{1 G}^{+} \alpha_{1} k_{1 G}-\beta_{1 P}^{-} \alpha_{1} k_{1 P}-c_{1}-c_{s}, \\
T= & \tau_{1 P} \alpha_{2} k_{2 P}+\tau_{1 G} \alpha_{2} k_{2 G}-f_{s}-f_{t}, \\
P= & -f_{t},
\end{aligned}
$$

$$
\begin{aligned}
A^{\prime}= & \mathrm{KS} \quad\left(\begin{array}{cc}
R^{\prime} & S^{\prime} \\
T^{\prime} & P^{\prime}
\end{array}\right), \\
& \mathrm{NKS} \\
R^{\prime}= & \Gamma_{2}\left[\alpha_{1}\left(k_{1 P}+k_{1 G}\right)+\alpha_{2}\left(k_{2 P}+k_{2 G}\right)\right] \\
& +\beta_{2 P}^{+} \alpha_{2} k_{2 P}+\beta_{2 G}^{+} \alpha_{2} k_{2 G}-\beta_{2 P}^{-} \alpha_{2} k_{2 P}-c_{2}, \\
S^{\prime}= & \beta_{2 P}^{+} \alpha_{2} k_{2 P}+\beta_{2 G}^{+} \alpha_{2} k_{2 G}-\beta_{2 P}^{-} \alpha_{2} k_{2 P}-c_{2}-c_{s}, \\
T^{\prime}= & \tau_{2 P} \alpha_{1} k_{1 P}+\tau_{2 G} \alpha_{1} k_{1 G}-f_{s}-f_{t}, \\
P^{\prime}= & -f_{t} .
\end{aligned}
$$

According to each player's pay-off, the universal dilemma strength is expressed as per equations (17)-(21), respectively, by following equation (14):

$$
\begin{gathered}
D_{g 1}^{\prime}=\frac{T-R}{R-P}=\frac{M}{Q}, D_{r 1}^{\prime}=\frac{P-S}{R-P}=\frac{N}{Q}, \\
M=\Gamma_{1}\left[\alpha_{1}\left(k_{1 P}+k_{1 G}\right)+\alpha_{2}\left(k_{2 P}+k_{2 G}\right)\right]-\tau_{1 P} \alpha_{2} k_{2 P} \\
-\tau_{1 G} \alpha_{2} k_{2 G}+\beta_{1 P}^{+} \alpha_{1} k_{1 P}+\beta_{1 G}^{+} \alpha_{1} k_{1 G}-\beta_{1 P}^{-} \alpha_{1} k_{1 P} \\
-c_{1}+f_{s}+f_{t}=-5,
\end{gathered}
$$

$N=\beta_{1 P}^{+} \alpha_{1} k_{1 P}+\beta_{1 G}^{+} \alpha_{1} k_{1 G}-\beta_{1 P}^{-} \alpha_{1} k_{1 P}-c_{1}-c_{s}+f_{t}=-$ (3),

$$
\begin{aligned}
Q= & \Gamma_{1}\left[\alpha_{1}\left(k_{1 P}+k_{1 G}\right)+\alpha_{2}\left(k_{2 P}+k_{2 G}\right)\right]+\beta_{1 P}^{+} \alpha_{1} k_{1 P} \\
& +\beta_{1 G}^{+} \alpha_{1} k_{1 G}-\beta_{1 P}^{-} \alpha_{1} k_{1 P}-c_{1} \\
& +f_{t}=(1)+(3)+\tau_{1 P} \alpha_{2} k_{2 P}+\tau_{1 G} \alpha_{2} k_{2 G}+c_{s}, \\
& D_{g^{\prime}}^{\prime}=\frac{T^{\prime}-R^{\prime}}{R^{\prime}-P^{\prime}}=\frac{M^{\prime}}{Q^{\prime}}, D_{r 2}^{\prime}=\frac{P^{\prime}-S^{\prime}}{R^{\prime}-P^{\prime}}=\frac{N^{\prime}}{Q^{\prime}}, \\
M^{\prime}= & \Gamma_{2}\left[\alpha_{1}\left(k_{1 P}+k_{1 G}\right)+\alpha_{2}\left(k_{2 P}+k_{2 G}\right)\right]-\tau_{2 P} \alpha_{1} k_{1 P} \\
& -\tau_{2 G} \alpha_{1} k_{1 G}+\beta_{2 P}^{+} \alpha_{2} k_{2 P}+\beta_{2 G}^{+} \alpha_{2} k_{2 G} \\
& -\beta_{2 P}^{-} \alpha_{2} k_{2 P}-c_{2}+f_{s}+f_{t}=- \text { (6), } \\
N^{\prime}= & \beta_{2 P}^{+} \alpha_{2} k_{2 P}+\beta_{2 G}^{+} \alpha_{2} k_{2 G}-\beta_{2 P}^{-} \alpha_{2} k_{2 P}-c_{2}-c_{s}+f_{t}=-(4), \\
Q^{\prime}= & \Gamma_{2}\left[\alpha_{1}\left(k_{1 P}+k_{1 G}\right)+\alpha_{2}\left(k_{2 P}+k_{2 G}\right)\right]+\beta_{2 P}^{+} \alpha_{2} k_{2 P} \\
& +\beta_{2 G}^{+} \alpha_{2} k_{2 G}-\beta_{2 P}^{-} \alpha_{2} k_{2 P}-c_{2}+f_{t}=(2) \\
& +(4)+\tau_{2 P} \alpha_{1} k_{1 P}+\tau_{2 G} \alpha_{1} k_{1 G}+c_{s} .
\end{aligned}
$$

(2) Evolutionary Equilibrium Point Analysis in Situation IX. In situation IX (as shown in Table 4 and Figure 4), the system can satisfy the following conditions: (1) $>0$, (2) $>0$, (3) $<0$, (4) $<0$, (5) $>0$, and (6) $>0$. Therefore, from equations (17)-(24), we can derive equations (25) and (26) when the system is under the conditions of equations (27) and (28) (for details, see Appendix B):

$$
D_{g 1}^{\prime}=\frac{M}{Q}<0, D_{r 1}^{\prime}=\frac{N}{Q}>0,
$$

$$
\begin{gathered}
D_{g^{2}}^{\prime}=\frac{M^{\prime}}{Q^{\prime}}<0, D_{r 2}^{\prime}=\frac{N^{\prime}}{Q^{\prime}}>0, \\
f_{t}>-\left\{\Gamma_{1}\left[\alpha_{1}\left(k_{1 P}+k_{1 G}\right)+\alpha_{2}\left(k_{2 P}+k_{2 G}\right)\right]\right. \\
\left.+\beta_{1 P}^{+} \alpha_{1} k_{1 P}+\beta_{1 G}^{+} \alpha_{1} k_{1 G}-\beta_{1 P}^{-} \alpha_{1} k_{1 P}-c_{1}\right\}, \\
f_{t}>-\left\{\Gamma_{2}\left[\alpha_{1}\left(k_{1 P}+k_{1 G}\right)+\alpha_{2}\left(k_{2 P}+k_{2 G}\right)\right]\right. \\
\left.+\beta_{2 P}^{+} \alpha_{2} k_{2 P}+\beta_{2 G}^{+} \alpha_{2} k_{2 G}-\beta_{2 P}^{-} \alpha_{2} k_{2 P}-c_{2}\right\} .
\end{gathered}
$$


In summary, in situation IX, when the system satisfies equations (27) and (28), we can obtain $D_{g 1}{ }^{\prime}<0, D_{r 1}{ }^{\prime}>0$, and $D_{g 2}^{\prime}<0, D_{r 2}^{\prime}>0$, which means internal equilibrium $\left(x^{*}, y^{*}\right)$ is unstable (bistable) and falls into region $1>x^{*}>0$ and $1>y^{*}>0$. In other words, $\{\mathrm{NKS}, \mathrm{NKS}\}$ and $\{\mathrm{KS}, \mathrm{KS}\}$ could both be the evolutionary stable strategy sets, that is, these two players either both choose NKS or KS in the evolutionary stable state (as shown in Figure 4).

\section{Simulation and Analysis}

To better understand how the variables influence the evolutionary stable state of the system, a simulation was conducted to analyze situation IX. In this case, the stable result of the system is affected by the initial probabilities of knowledge sharing for both players and the dynamic changes of the relevant parameters. Here, we simulate the evolutionary processes of the system with function ode 45 and the function plot in Matlab 2017, and replicator dynamic equations (7) and (8) are solved. The initial parameters are set as shown in Table 5. The parameters' initial values and their changes satisfy the following conditions: polynomials (1) $>0$, (2) $>0$, (3) $<0$, (4) $<0$, (5) $>0$, (6) $>0$; and $\beta_{1 P}^{+}>\beta_{1 G}^{+}$, $\beta_{2 P}^{+}>\beta_{2 G}^{+}, \tau_{1 P}<\tau_{1 G}, \tau_{2 P}<\tau_{2 G}$. Moreover, they coincide with equations (27) and (28). Subsequently, the results for the main parameters influencing the evolutionary game are tested separately and concurrently, as shown in Figures 5-12 . The $x$-axis in Figures 5-12 represents the time, while the $y$ axis represents the probability of choosing KS.

\subsection{Comparison of the Effects of Different Punishment} Mechanisms on Knowledge Sharing. Here, no punishment, second-party punishment, third-party punishment, and mixed punishment are compared to identify which one facilitates knowledge sharing best. Additionally, simultaneously adopting second-and-third-party punishment as a mixed punishment is considered. Further, no punishment can be considered as reference. The simulation results regarding the different punishment mechanisms are as follows.

Figure 5 characterizes the dynamical evolutionary processes of the probability of knowledge sharing for four punishment types: no punishment $\left(y_{1}\right)$, second-party punishment $\left(y_{2}\right)$, third-party punishment $\left(y_{3}\right)$, and mixed punishment $\left(y_{4}\right)$. Figure 5(a) shows the results for player1, Figure 5(b) the results for player2, and Figure 5(c) a combination of Figures 5(a) and 5(b), that is, the results for both player1 and player2. As we can see from Figures 5(a) and 5 (b), $y_{2}, y_{3}$, and $y_{4}$ (red, yellow, and purple curves) all converge to strategy set $\{\mathrm{KS}, \mathrm{KS}\}$, which means both players eventually choose to share when any punishment is implemented. However, the speeds of convergence are different, while $y_{2}$ is the slowest, while $y_{3}$ and $y_{4}$ have similar speeds and are both much faster than $y_{2}$. That is, the third-party and mixed punishments have similar promoting effects on knowledge sharing and are both more significant than second-party punishment. Therefore, the mixed punishment is not a good choice to promote knowledge sharing, because punishment is a negative incentive, which may cause dissatisfaction among employees. As such, it is not suitable for the organization to adopt excessive punishment to promote the occurrence of knowledge sharing behavior. Generally, the best choice for an organization is to implement third-party punishment to increase the knowledge contribution.

4.2. Further Analysis of the Impacts of Second-and Third-Party Punishments on Knowledge Sharing. From the above discussion, third-party punishment can lead to a significantly better knowledge sharing performance compared to secondparty punishment. To better understand the mechanisms of second- and third-party punishments on knowledge sharing, some questions still need to be explored, such as the relationships between second-party punishment cost, penalty of second-party punishment, penalty of third-party punishment, and knowledge sharing. Figure 6 represents the results of these analyses.

Figure 6 aims to describe the dynamical evolutionary processes of the probability of knowledge sharing for five situation types: (1) with second-party punishment, $c_{s}=1$, $f_{s}=2$; (2) with second-party punishment, $c_{s}=0.5, f_{s}=2$; (3) with second-party punishment, $c_{s}=1, f_{s}=3$; (4) with third-party punishment, $f_{t}=2$; and (5) with third-party punishment, $f_{t}=3$. Figure $6(\mathrm{a})$ shows the results for player1, Figure 6(b) for player2, and Figure 6(c) shows a combination of Figures 6(a) and 6(b), that is, the results for both player1 and player2.

First, we discuss the relationship between second-party punishment cost and knowledge sharing. In Figures 6(a) and $6(\mathrm{~b})$, the red curve is above the blue curve, which means the effect of second-party punishment on knowledge sharing is enhanced when the second-party punishment cost decreases from $c_{s}=1$ to $c_{s}=0.5$, while keeping the other parameters constant. Therefore, as the second-party punishment cost is reduced, the value of saddle point $D\left(x^{*}, y^{*}\right)$ decreases, as does the OADB area, and the converging possibility of the system to stable point $(1,1)$ increases. That is, the probability of choosing KS for both players increases, and the knowledge sharing behavior is promoted. As a result, the second-party punishment cost is negatively correlated with the knowledge sharing behavior.

Second, we discuss the relationship between the penalty of second-party punishment and knowledge sharing. In Figures 6(a) and 6(b), the yellow curve is above the blue curve, which means the promotion effect of second-party punishment on knowledge sharing is increased when raising the penalty of second-party punishment from $f_{s}=2$ to $f_{s}=3$, while keeping the other parameters constant. Therefore, as the penalty of secondparty punishment increases, the value of saddle point $D\left(x^{*}, y^{*}\right)$ decreases, as does the OADB area, while the converging possibility of the system to stable point $(1,1)$ improves. That is, the probability of choosing KS for both players increases and the knowledge sharing behavior is enhanced. As a result, the penalty of second-party punishment is positively correlated with the knowledge sharing behavior. 
TABLE 5: Initial parameter values.

\begin{tabular}{lcccccccccccc}
\hline Parameter & $k_{1 P}$ & $k_{1 G}$ & $k_{2 P}$ & $k_{2 G}$ & $\alpha_{1}$ & $\alpha_{2}$ & $\beta_{1 P}^{+}$ & $\beta_{1 G}^{+}$ & $\beta_{2 P}^{+}$ & $\beta_{2 G}^{+}$ & $\beta_{1 P}^{-}$ & $\beta_{2 P}^{-}$ \\
Value & 60 & 80 & 50 & 70 & 0.04 & 0.06 & 0.5 & 0.05 & 0.4 & 0.04 & 0.7 & 0.8 \\
\hline Parameter & $\tau_{1 P}$ & $\tau_{2 P}$ & $\tau_{1 G}$ & $\tau_{2 G}$ & $\Gamma_{1}$ & $\Gamma_{2}$ & $c_{1}$ & $c_{2}$ & $c_{s}$ & $f_{s}$ & $f_{t}$ & 2 \\
Value & 0.3 & 0.2 & 0.6 & 0.5 & 1.2 & 1.1 & 5 & 6 & 1 & 2 \\
\hline
\end{tabular}

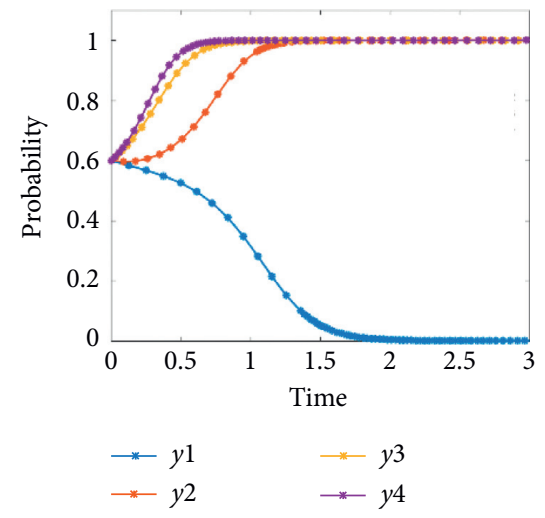

(a)

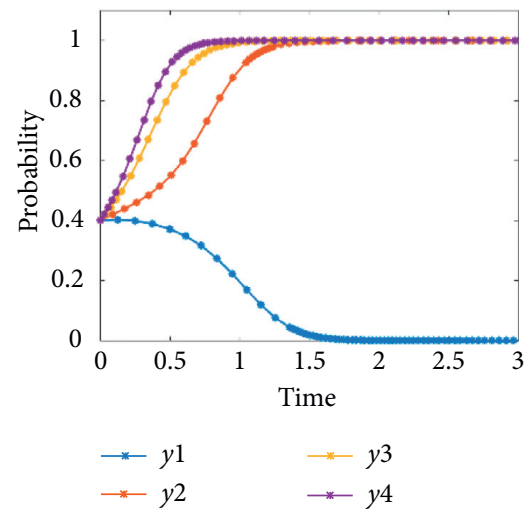

(b)

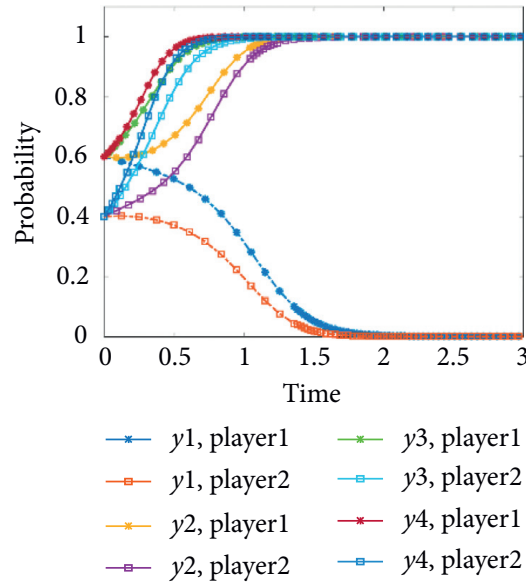

(c)

FIGURE 5: The dynamical evolutionary processes of the probability of knowledge sharing for four punishment types: no punishment $\left(y_{1}\right)$, second-party punishment $\left(y_{2}\right)$, third-party punishment $\left(y_{3}\right)$, and mixed punishment $\left(y_{4}\right)$. (a) The results for player1; (b) the results for player2; and (c) the results for both players.

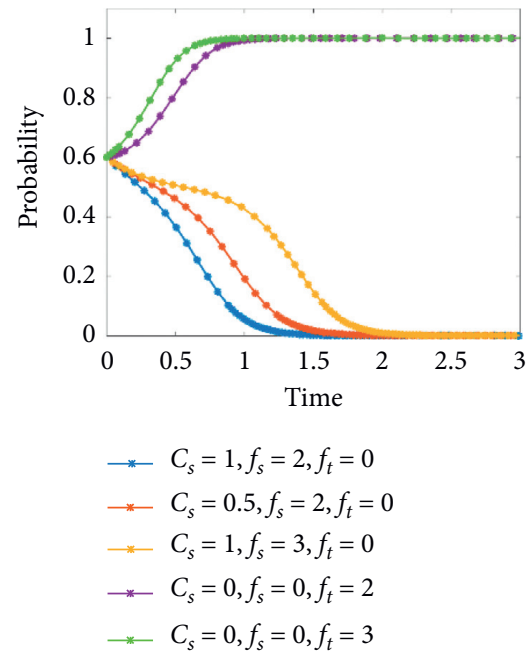

(a)

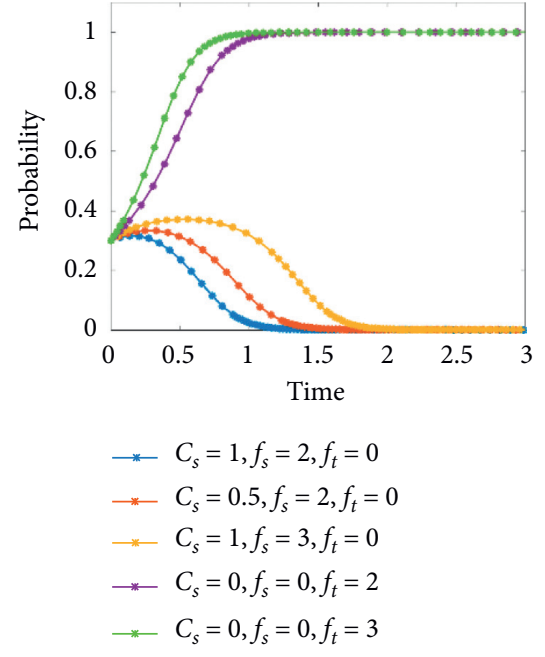

(b)

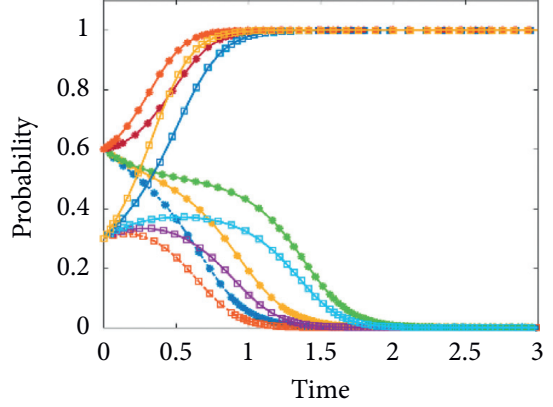

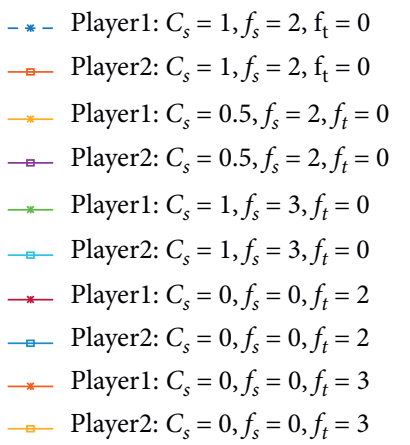

(c)

FIgURE 6: The dynamical evolutionary processes of the probability of knowledge sharing for five types of situations: (1) with second-party punishment, $c_{s}=1, f_{s}=2$; (2) with second-party punishment, $c_{s}=0.5, f_{s}=2$; (3) with second-party punishment, $c_{s}=1, f_{s}=3$; (4) with third-party punishment, $f_{t}=2$; (5) with third-party punishment, $f_{t}=3$. (a) The results for player1; (b) the results for player2; and (c) the results for both players. 


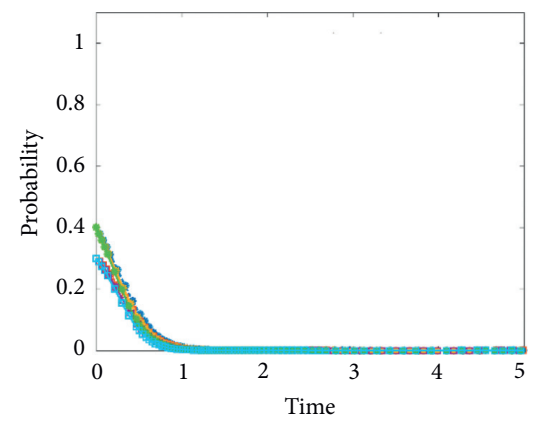

$$
\begin{aligned}
& -\because-\text { Player1: } \beta_{1 c}^{+}=0.6, \beta_{2 c}^{+}=0.5, \beta_{1 c}^{-}=0.5, \beta_{2 c}^{-}=0.4 \\
& -\Xi-\text { Player2: } \beta_{1 c}^{+}=0.6, \beta_{2 c}^{+}=0.5, \beta_{1 c}^{-}=0.5, \beta_{2 c}^{-}=0.4 \\
& \longrightarrow \text { Player1: } \beta_{1 c}^{+}=0.5, \beta_{2 c}^{+}=0.4, \beta_{1 c}^{-}=0.5, \beta_{2 c}^{-}=0.4 \\
& \longrightarrow \text { Player2: } \beta_{1 c}^{+}=0.5, \beta_{2 c}^{+}=0.4, \beta_{1 c}^{-}=0.5, \beta_{2 c}^{-}=0.4 \\
& \longrightarrow \text { Player1: } \beta_{1 c}^{+}=0.5, \beta_{2 c}^{+}=0.4, \beta_{1 c}^{-}=0.6, \beta_{2 c}^{-}=0.5 \\
& \square \text { Player2: } \beta_{1 c}^{+}=0.5, \beta_{2 c}^{+}=0.4, \beta_{1 c}^{-}=0.6, \beta_{2 c}^{-}=0.5
\end{aligned}
$$

(a)

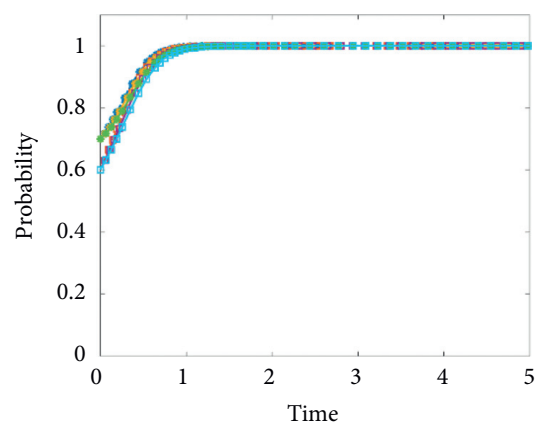

- * Player $1: \beta_{1 c}^{+}=0.6, \beta_{2 c}^{+}=0.5, \beta_{1 c}^{-}=0.5, \beta_{2 c}^{-}=0.4$

- $\boxminus$ - Player2: $\beta_{1 c}^{+}=0.6, \beta_{2 c}^{+}=0.5, \beta_{1 c}^{-}=0.5, \beta_{2 c}^{-}=0.4$

- Player $1: \beta_{1 c}^{+}=0.5, \beta_{2 c}^{+}=0.4, \beta_{1 c}^{-}=0.5, \beta_{2 c}^{-}=0.4$

$\square$ Player2: $\beta_{1 c}^{+}=0.5, \beta_{2 c}^{+}=0.4, \beta_{1 c}^{-}=0.5, \beta_{2 c}^{-}=0.4$

$\longrightarrow$ Player1: $\beta_{1 c}^{+}=0.5, \beta_{2 c}^{+}=0.4, \beta_{1 c}^{-}=0.6, \beta_{2 c}^{-}=0.5$

$\square$ Player2: $\beta_{1 c}^{+}=0.5, \beta_{2 c}^{+}=0.4, \beta_{1 c}^{-}=0.6, \beta_{2 c}^{-}=0.5$

(b)

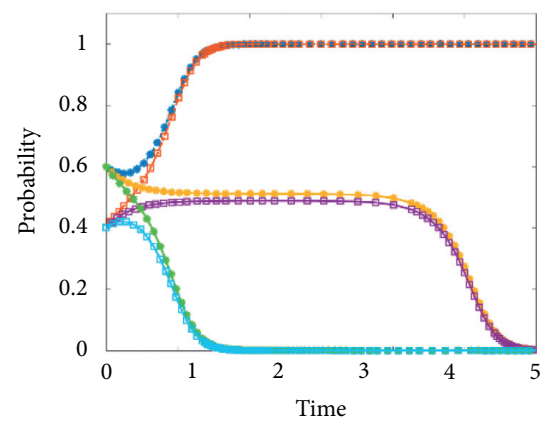

- *- Player1: $\beta_{1 c}^{+}=0.6, \beta_{2 c}^{+}=0.5, \beta_{1 c}^{-}=0.5, \beta_{2 c}^{-}=0.4$

- - Player2: $\beta_{1 c}^{+}=0.6, \beta_{2 c}^{+}=0.5, \beta_{1 c}^{-}=0.5, \beta_{2 c}^{-}=0.4$

—-Player1: $\beta_{1 c}^{+}=0.5, \beta_{2 c}^{+}=0.4, \beta_{1 c}^{-}=0.5, \beta_{2 c}^{-}=0.4$

$\simeq$ Player2: $\beta_{1 c}^{+}=0.5, \beta_{2 c}^{+}=0.4, \beta_{1 c}^{-}=0.5, \beta_{2 c}^{-}=0.4$

*- Player1: $\beta_{1 c}^{+}=0.5, \beta_{2 c}^{+}=0.4, \beta_{1 c}^{-}=0.6, \beta_{2 c}^{-}=0.5$

$\because$ Player2: $\beta_{1 c}^{+}=0.5, \beta_{2 c}^{+}=0.4, \beta_{1 c}^{-}=0.6, \beta_{2 c}^{-}=0.5$

(c)

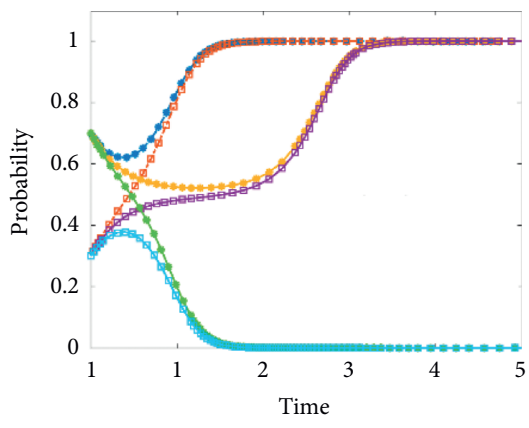

-*- Player1: $\beta_{1 c}^{+}=0.6, \beta_{2 c}^{+}=0.5, \beta_{1 c}^{-}=0.5, \beta_{2 c}^{-}=0.4$

- $\Xi-$ Player2: $\beta_{1 c}^{+}=0.6, \beta_{2 c}^{+}=0.5, \beta_{1 c}^{-}=0.5, \beta_{2 c}^{-}=0.4$

— Player1: $\beta_{1 c}^{+}=0.5, \beta_{2 c}^{+}=0.4, \beta_{1 c}^{-}=0.5, \beta_{2 c}^{-}=0.4$

$\square$ Player2: $\beta_{1 c}^{+}=0.5, \beta_{2 c}^{+}=0.4, \beta_{1 c}^{-}=0.5, \beta_{2 c}^{-}=0.4$

$\longrightarrow$ Playerl: $\beta_{1 c}^{+}=0.5, \beta_{2 c}^{+}=0.4, \beta_{1 c}^{-}=0.6, \beta_{2 c}^{-}=0.5$

$\square$ Player2: $\beta_{1 c}^{+}=0.5, \beta_{2 c}^{+}=0.4, \beta_{1 c}^{-}=0.6, \beta_{2 c}^{-}=0.5$

(d)

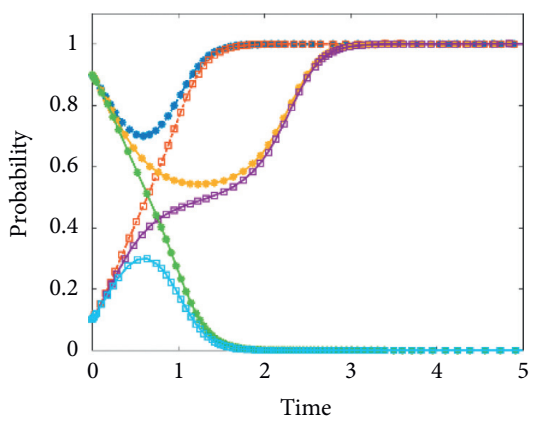

- *- Player $1: \beta_{1 c}^{+}=0.6, \beta_{2 c}^{+}=0.5, \beta_{1 c}^{-}=0.5, \beta_{2 c}^{-}=0.4$

- - Player2: $\beta_{1 c}^{+}=0.6, \beta_{2 c}^{+}=0.5, \beta_{1 c}^{-}=0.5, \beta_{2 c}^{-}=0.4$

— Player 1: $\beta_{1 c}^{+}=0.5, \beta_{2 c}^{+}=0.4, \beta_{1 c}^{-}=0.5, \beta_{2 c}^{-}=0.4$

$\square$ Player2: $\beta_{1 c}^{+}=0.5, \beta_{2 c}^{+}=0.4, \beta_{1 c}^{-}=0.5, \beta_{2 c}^{-}=0.4$

— Player1: $\beta_{1 c}^{+}=0.5, \beta_{2 c}^{+}=0.4, \beta_{1 c}^{-}=0.6, \beta_{2 c}^{-}=0.5$

$\because$ Player2: $\beta_{1 c}^{+}=0.5, \beta_{2 c}^{+}=0.4, \beta_{1 c}^{-}=0.6, \beta_{2 c}^{-}=0.5$

(e)

FIgURE 7: The dynamical evolutionary processes of the probability of knowledge sharing for three types of situations: (1) when the status conflict advantage is greater than the disadvantage $\left(\beta_{1 P}^{+}=0.6>\beta_{1 P}^{-}=0.5, \beta_{2 P}^{+}=0.5>\beta_{2 P}^{-}=0.4\right)$; (2) when the status conflict advantage is equal to the disadvantage $\left(\beta_{1 P}^{+}=\beta_{1 P}^{-}=0.5, \beta_{2 P}^{+}=\beta_{2 P}^{-}=0.4\right)$; (3) when the status conflict advantage is below the disadvantage $\left(\beta_{1 P}^{+}=0.5<\beta_{1 P}^{-}=0.6, \beta_{2 P}^{+}=0.4<\beta_{2 P}^{-}=0.5\right)$. (a) The results for when the initial willingness of knowledge sharing of the two players are, respectively, set as $x=0.4, y=0.3$; (b) the results for when the initial willingness of knowledge sharing of the two players are, respectively, set as $x=0.7, y=0.6$; (c) the results for when the initial willingness of knowledge sharing of the two players are, respectively, set as $x=0.6, y=0.4$; (d) the results for when the initial willingness of knowledge sharing of the two players are, respectively, set as $x=0.7, y=0.3$; and (e) the results for when the initial willingness of knowledge sharing of the two players are, respectively, set as $x=0.9, y=0.1$.

Third, we test the relationship between the penalty of third-party punishment and knowledge sharing. In Figures 6(a) and 6(b), the green curve is above the purple curve, which means the promotion effect of third-party punishment on knowledge sharing is encouraged when increasing the penalty of third-party punishment from $f_{t}=$ 2 to $f_{t}=3$, while keeping the other parameters constant. Therefore, as the penalty of third-party punishment increases, the value of saddle point $D\left(x^{*}, y^{*}\right)$ decreases, as does area $\mathrm{OADB}$, and the converging possibility of the system to stable point $(1,1)$ increases. That is, the probability of choosing KS increases for both players, and the knowledge sharing behavior is promoted.

Finally, we further compare between the positive influence of second- and third-party punishments on knowledge sharing. In Figures 6(a) and 6(b), the purple curve is above the yellow one, which is in turn above the red curve. That is, the purple curve can reach the best effect of improving knowledge sharing, while the red curve's is the weakest. Even when the second-party punishment cost is low $\left(c_{s}=0.5\right.$, the red curve) or the penalty of second-party punishment is high $\left(f_{s}=3\right.$, the yellow curve), the 


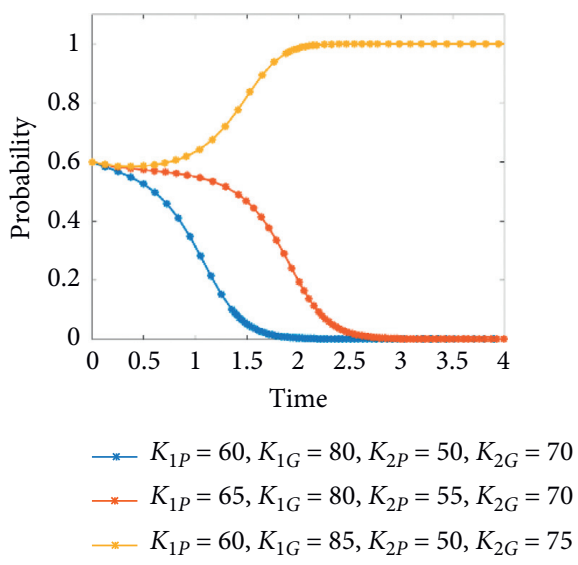

(a)

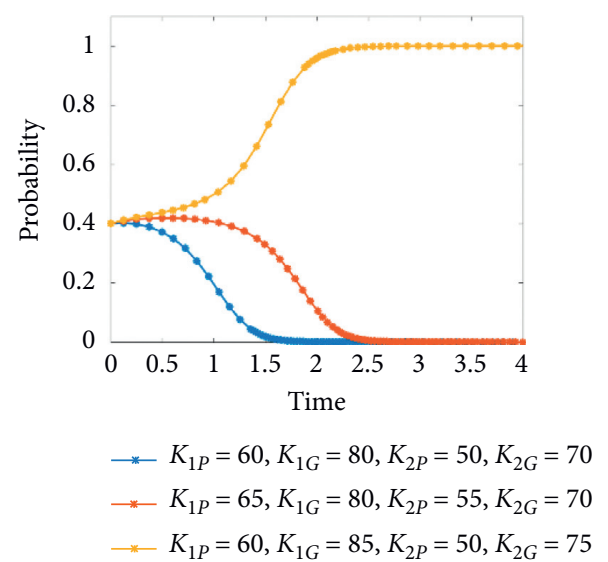

(b)

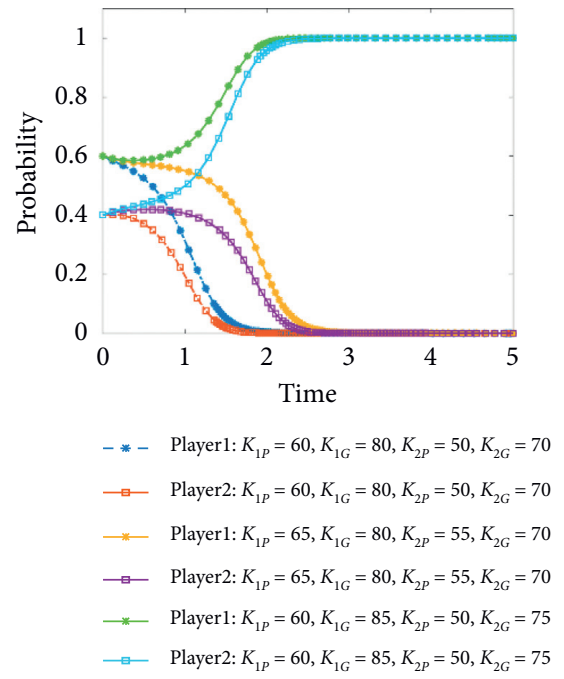

(c)

Figure 8: The dynamical evolutionary processes of the probability of knowledge sharing for different professional and general knowledge capacity: (1) when professional knowledge capacity increases from $K_{1 P}=60, K_{2 P}=50$ to $K_{1 P}=65$, and $K_{2 P}=55$; (2) when general knowledge capacity increases from $K_{1 G}=80, K_{2 G}=70$ to $K_{1 G}=85$, and $K_{2 G}=75$. (a) The results for player1; (b) the results for player2; and (c) the results for both players.

promotion effect of the second-party punishment on knowledge sharing is not as significant as that of third-party punishment ( $f_{t}=2$, the purple curve). This further demonstrates that either second- or third-party punishment can facilitate knowledge sharing behavior, but the knowledge contribution increases more noticeably with third-party punishment than with second-party punishment.

\subsection{Effects of Status Conflict and Initial Sharing Willingness on} Knowledge Sharing. Sharing knowledge brings both benefits and losses to individuals. Therefore, we concurrently consider the status conflict advantage and disadvantage. Figure 7 shows the dynamical evolutionary processes of the probability of knowledge sharing for three types of situations: (1) when the status conflict advantage is greater than the disadvantage, (2) when the status conflict advantage is equal to the disadvantage, and (3) when the status conflict advantage is lower than the disadvantage.
In Figures 7(a) and 7(b), when both players have weak/ strong initial willingness toward knowledge sharing $(x=0.4, y=0.3 / x=0.7, y=0.6$, we set 0.5 as the critical value), the stable equilibrium strategy of the system is $\{\mathrm{NKS}$, $\mathrm{NKS}\} /\{\mathrm{KS}, \mathrm{KS}\}$, regardless whether the status conflict advantage or disadvantage is stronger. That is, the initial values of $x$ and $y$ have significant impacts on knowledge sharing. Below, we further discuss the relationship between the initial state and knowledge sharing behavior.

However, when two players have initially different opinions on knowledge sharing, the status conflict advantage and disadvantage play important roles in knowledge sharing. From Figures 7(c)-7(e), when the status conflict advantage is greater than the disadvantage $\left(\beta_{1 P}^{+}=0.6>\beta_{1 P}^{-}=0.5\right.$, $\left.\beta_{2 P}^{+}=0.5>\beta_{2 P}^{-}=0.4\right)$, the stable point of the system is $(1,1)$ (red and blue curves), which means both players eventually choose KS and the free riding behavior disappears. By contrast, when the status conflict advantage is below the disadvantage $\left(\beta_{1 P}^{+}=0.5<\beta_{1 P}^{-}=0.6, \beta_{2 P}^{+}=0.4<\beta_{2 P}^{-}=0.5\right)$, 


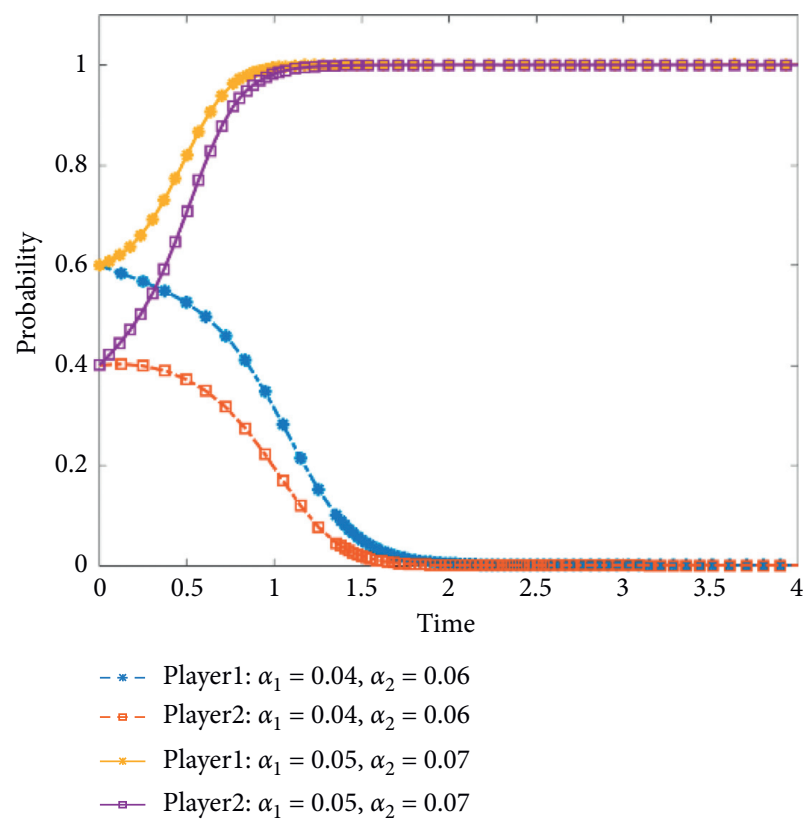

FIGURE 9: Evolutionary game phase diagram of the system (when $\alpha_{1}$ and $\alpha_{2}$ improve).

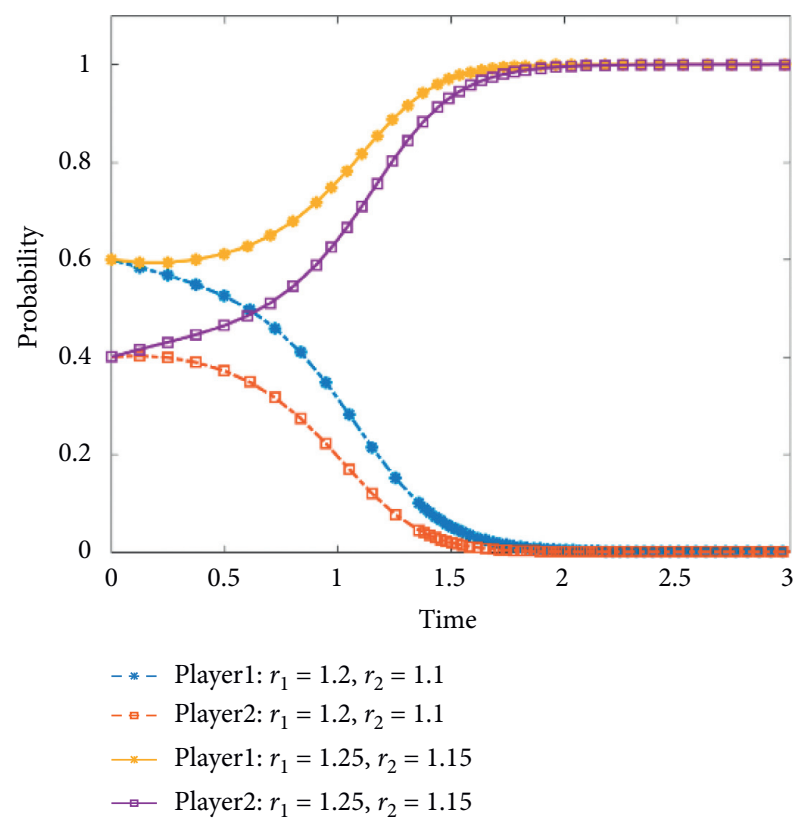

FIGURE 10: Evolutionary game phase diagram of the system (when $r_{1}$ and $r_{2}$ improve).

the stable point of the system is $(0,0)$ (light blue and green curves), which means both players finally choose NKS and the knowledge sharing behavior disappears.

Furthermore, when the status conflict advantage is equal to the disadvantage $\left(\beta_{1 P}^{+}=\beta_{1 P}^{-}=0.5, \beta_{2 P}^{+}=\beta_{2 P}^{-}=0.4\right)$, one player can influence the other one to share knowledge if the former has initially a strong willingness for sharing. In Figure $7(\mathrm{c})$ (purple and yellow curves), both players' knowledge sharing willingness is close to the critical value of

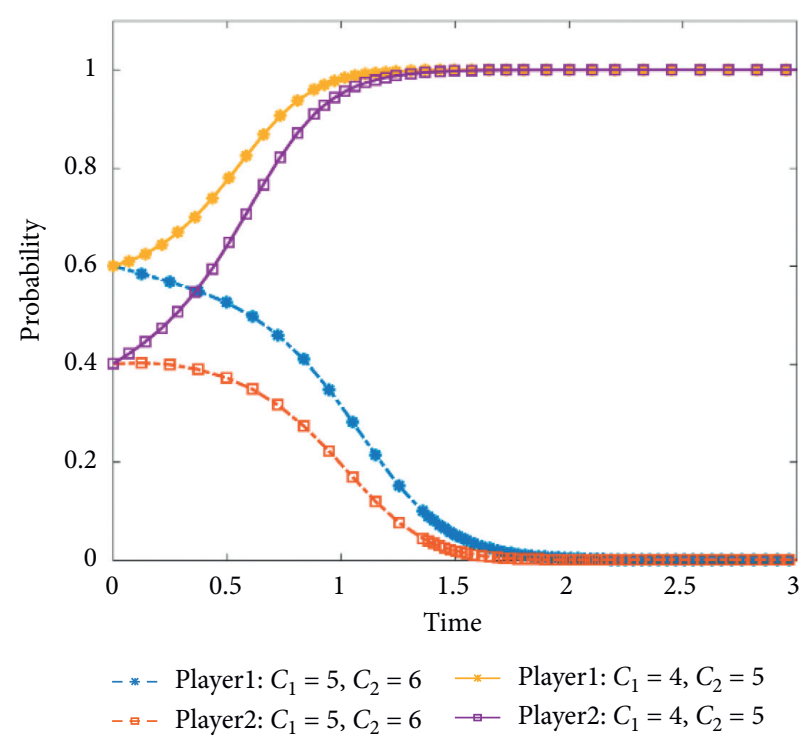

FIGURE 11: Evolutionary game phase diagram of the system (when $C_{1}$ and $C_{2}$ decrease).

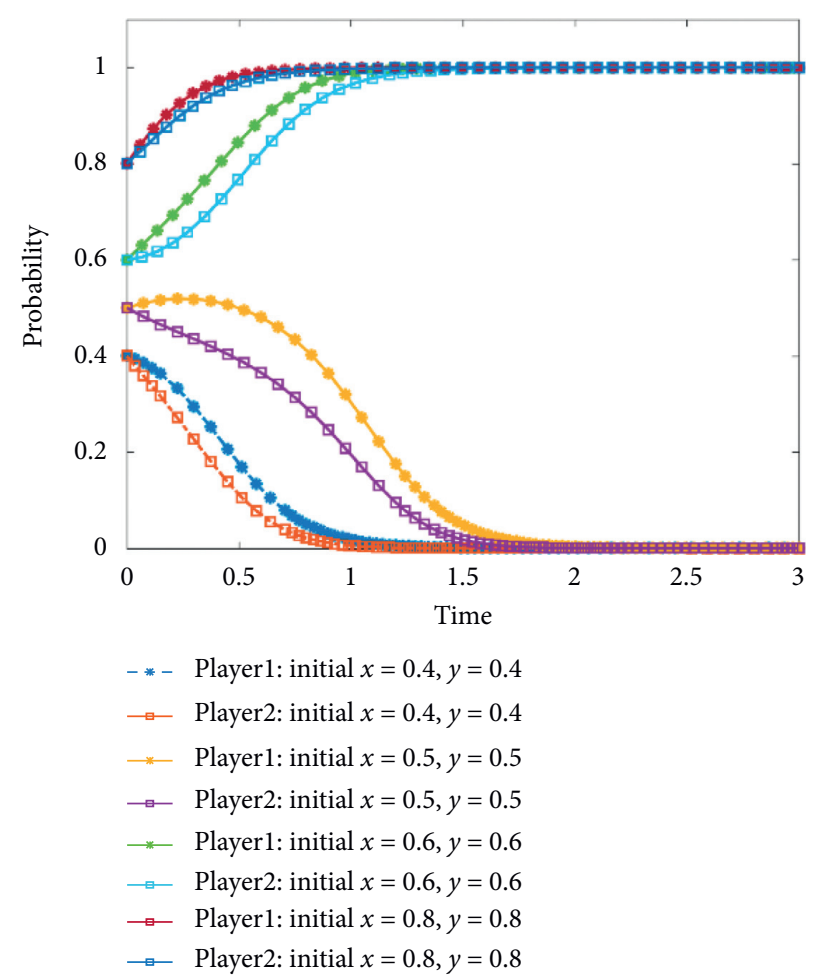

Figure 12: Evolutionary game phase diagram of the system for different initial states.

$0.5(x=0.6, y=0.4)$; that is, neither of them has a strong opinion to contributing knowledge. Therefore, the system converges to stable point $(0,0)$ after a period. In other words, both players would eventually choose NKS in this case. Nevertheless, the situation changes if one player has initially a strong opinion on sharing knowledge. Figures $7(\mathrm{~d})$ and 7 (e) (purple and yellow curves) show the dynamical evolutionary results when we set $x=0.7, y=0.3$ and 
$x=0.9, y=0.1$, respectively (we draw the same conclusion when we set $x=0.3, y=0.7$ and $x=0.1, y=0.9$ ). In these situations, one player has a strong willingness to share knowledge, while the other would rather hoard it. Interestingly, the player who has a high tendency to hoard knowledge is influenced by the other one. Consequently, both players would choose KS and the system reaches stable state $(1,1)$. Generally, the player with a strong opinion on sharing knowledge has a powerful impact on the other player, who has a weak opinion on sharing and can lead him/ her to ultimately share knowledge.

\subsection{Effects of Knowledge Capacity on Knowledge Sharing.} The knowledge capacity denotes the maximal quantity of sharable knowledge and has a strong influence on the knowledge sharing behavior. Figure 8 describes the dynamical evolutionary processes of the knowledge sharing probability for different professional and general knowledge capacities. Specifically, Figure 8(a) shows the results for player1, Figure 8(b) for player2, and Figure 8(c) a combination of Figures $8(\mathrm{a})$ and $8(\mathrm{~b})$, that is, the results for both player1 and player2. In Figures 8(a) and 8(b), the red and yellow curves are both above the blue curve, which means the knowledge sharing behavior is promoted when the professional knowledge capacity increases from $K_{1 P}=60$, $K_{2 P}=50$ to $K_{1 P}=65, K_{2 P}=55$ when the general knowledge capacity increases from $K_{1 G}=80, K_{2 G}=70$ to $K_{1 G}=85$, $K_{2 G}=75$, while keeping the other parameters constant. Therefore, as the knowledge capacity increases, the value of saddle point $D\left(x^{*}, y^{*}\right)$ decreases, as does area OADB, and the converging possibility of the system to stable point $(1,1)$ increases. That is, the probability of choosing KS increases for both players and knowledge sharing behavior is enhanced. As a result, the knowledge capacity is positively correlated with the knowledge sharing behavior.

4.5. Other Parameters. Guanxi is a significant parameter influencing knowledge sharing behavior. Figure 9 illustrates the simulation results of the dynamical evolutionary processes of the probability of knowledge sharing when guanxi changes. From Figure 9, the evolutionary stable point alters from $(0,0)$ to $(1,1)$ when guanxi increases from $\alpha_{1}=0.04$, $\alpha_{2}=0.06$ to $\alpha_{1}=0.05, \alpha_{2}=0.07$, while keeping the other parameters constant. Therefore, as guanxi increases, the value of saddle point $D\left(x^{*}, y^{*}\right)$ decreases, as does area $\mathrm{OADB}$, and the converging possibility of the system to the stable point $(1,1)$ increases. That is, the probability of choosing KS increases for both players and the knowledge sharing behavior improves. As a result, guanxi is positively correlated with the knowledge sharing behavior.

The synergy revenue coefficient of KS has a strong influence on the knowledge sharing behavior. Figure 10 expresses the simulation results of the dynamical evolutionary processes of the probability of knowledge sharing when the synergy revenue coefficient changes. From Figure 10, the evolutionary stable point changes from $(0,0)$ to $(1,1)$ when the synergy revenue coefficient increases from $\Upsilon_{1}=1.2, \Upsilon_{2}=$ 1.1 to $\Upsilon_{1}=1.25, \Upsilon_{2}=1.15$ while keeping the other parameters constant. Therefore, as the synergy revenue coefficient increases, the value of saddle point $D\left(x^{*}, y^{*}\right)$ decreases, as does area $\mathrm{OADB}$, and the converging possibility of the system to the stable point $(1,1)$ increases. Therefore, the probability of choosing KS increases for both players and the knowledge sharing behavior is enhanced. As a result, the synergy revenue coefficient is positively correlated with the knowledge sharing behavior.

The knowledge sharing cost has a negative influence on the knowledge sharing behavior. Figure 11 shows the simulation results of the dynamical evolutionary processes of the probability of knowledge sharing when the knowledge sharing cost changes. From Figure 11, the evolutionary stable point alters from $(0,0)$ to $(1,1)$ when the knowledge sharing cost decreases from $C_{1}=5, C_{2}=6$ to $C_{1}=4, C_{2}=5$ while keeping the other parameters constant. Therefore, as the knowledge sharing cost decreases, the value of saddle point $D\left(x^{*}, y^{*}\right)$ also decreases along with area OADB, while the converging possibility of the system to stable point $(1,1)$ increases. That is, the probability of choosing KS increases for both players and the knowledge sharing behavior is promoted. As a result, the knowledge sharing cost is negatively correlated with the knowledge sharing behavior.

The results of the dynamical evolutionary process are affected by the initial system state. Figure 12 shows the different results for the different initial probabilities of knowledge sharing, while keeping the other parameters constant. As shown in Figure 12, when the initial values of $x$ and $y$ are both set as 0.4 or 0.5 , the evolutionary stable point is $(0,0)$. That is, both players would eventually choose NKS and the sharing knowledge behavior disappears. Nevertheless, the evolutionary stable point changes from $(0,0)$ to $(1,1)$ when the initial values of $x$ and $y$ are both increased to 0.6 or 0.8 . That is, both players would finally choose KS and the free-riding behavior disappears. Therefore, the possibility of the system to converge to stable point $(1,1)$ improves as the initial values of $x$ and $y$ increase, which means the probability of choosing KS increases for both players, and knowledge sharing behavior is facilitated when $x$ and $y$ are enhanced. As a result, the initial values of $x$ and $y$ have positive effects on the knowledge sharing behavior.

\section{Discussion}

5.1. Conclusions. Altruistic punishment helps maintain and consolidate social norms and sustain cooperation in societies and is present across a highly diverse range of human populations, while emerging in diverse patterns for each population $[20,22,23]$. Altruistic punishment includes second- and third-party punishments. In most extant studies, second- and third-party punishments are discussed together and generally regarded as altruistic punishment. However, although both of them are altruistic behaviors, there are some differences between. It is thus necessary conduct research on those differences to better understand altruistic behavior. Based on prior research, we analyzed knowledge sharing behavior by introducing an evolutionary game model with second- and third-party punishment mechanisms to determine which one improves knowledge 
sharing within an organization best. Using a Matlab programming simulation, we can also better understand the effects of altruistic punishment on knowledge sharing and the differences between second- and third-party punishments.

Moreover, we conducted a more in-depth investigation of the relationship between status conflict and knowledge sharing. A company's employees not only have the need to pursue tangible resources, such as money, but also require intangible resources, such as status [29]. As such, employees will use sharing knowledge as an interpersonal strategy to gain recognition and respect to improve their social prestige and obtain power and status [32, 33]. However, knowledge sharing not only brings employees advantages in the status conflict but also leads to disadvantages. Without the control of professional knowledge, the knowledge holder loses the competitive advantage from knowledge, which directly threatens his/her power and status in the organization [11,37]. We discuss the influence of status conflict on the knowledge sharing behavior by concurrently considering the status conflict advantage and disadvantage.

Using a combination of evolutionary game modeling and simulation, the following conclusions were obtained. (1) Second-party, third-party, and mixed punishments (i.e., simultaneously adopting second- and third-party punishments) were compared to identify which one facilitates knowledge sharing best. We can conclude that knowledge sharing behavior is enhanced when any of the punishment mechanisms is implemented. However, the degrees of improvement are different: third-party punishment and mixed punishment have similar promoting effects on knowledge sharing, both being more significant than that of secondparty punishment. In other words, the appropriate choice for an organization is to implement third-party punishment to increase the knowledge contribution because punishment is a negative incentive, which may cause dissatisfaction to employees. Therefore, it is not suitable for the organization to adopt excessive punishment to promote knowledge sharing behaviors. (2) The second-party punishment cost is negatively correlated with the knowledge sharing behavior and the penalties of second- or third-party punishments are positively correlated with knowledge sharing. Furthermore, even when the second-party punishment cost is low or the penalty of second-party punishment is high, the promotion effect of second-party punishment on knowledge sharing is not as significant as that of third-party punishment. This further demonstrates that either second- or third-party punishment can facilitate knowledge sharing behaviors, but the knowledge contribution increases more noticeably with third-party punishment rather than with second-party punishment. (3) When both players have weak/strong initial willingness toward knowledge sharing, the stable equilibrium strategy set of the system is $\{\mathrm{NKS}, \mathrm{NKS}\} /\{\mathrm{KS}, \mathrm{KS}\}$, regardless of whether the status conflict advantage or disadvantage is stronger. However, the situation changes when the two players have different initial opinions on knowledge sharing, as the status conflict advantage and disadvantage play important roles in knowledge sharing. When the status conflict advantage is greater than the disadvantage, both players eventually choose KS and the free-riding behavior disappears. By contrast, when the status conflict advantage is lower than the disadvantage, both players finally choose NKS and knowledge sharing behavior disappears. Interestingly, when the status conflict advantage is equal to the disadvantage, the player with a strong opinion on sharing knowledge has a powerful impact on the other player, who has a weak opinion on sharing, and can lead the second player to ultimately share knowledge. (4) The general and professional knowledge capacity, guanxi, and synergy revenue coefficient are all positively correlated with the knowledge sharing behavior. Meanwhile, the knowledge sharing cost has a negative influence on the knowledge sharing behavior. Moreover, the results of the dynamical evolutionary process are affected by the initial state of the system, that is, the initial values of $x$ and $y$ have positive effects on the knowledge sharing behavior.

5.2. Managerial Implications. Based on the above conclusions, the following management suggestions are proposed to promote knowledge sharing in an organization. First, implementing effective punishment mechanisms can effectively reduce free riding and promote knowledge sharing behavior. A suitable choice for an organization is to adopt third-party punishment for increasing the knowledge contribution; however, it is not necessary to excessively punish the free rider, since punishment is a negative incentive that may cause dissatisfaction among employees. Furthermore, third-party punishment can be combined with the need of employee self-realization. For example, the opportunities of getting promoted or obtaining better work arrangements could be appropriately reduced for the free rider.

Second, an open and caring team climate can encourage knowledge sharing for several reasons as follows. (1) It helps increase the status conflict advantage of employees by sharing general and professional knowledge and can also decrease the status conflict disadvantage due to sharing professional knowledge. Additionally, managers could elicit beliefs among their employees that their efforts of sharing knowledge are distinguishable and valuable, so that any incurred cost is not wasted. (2) It can encourage the employees who have strong opinions on sharing knowledge to lead the ones with a low tendency of sharing knowledge, since the former have a strong influence on the latter. (3) It encourages the development of guanxi and, consequently, the exchange of learning and knowledge. (4) It reduces the cost of communication between employees and improves their satisfaction to enhance the synergy revenue coefficient of KS. (5) It can encourage knowledge sharing behaviors by improving the initial probabilities of employees choosing knowledge sharing.

Finally, it is necessary to establish a sustainable training system. On one hand, a good learning atmosphere can encourage continuity in communication to enhance employees' knowledge absorptive capacities. On the other hand, it can urge employees to improve their skills and increase their knowledge capacity, which has a positive influence on 
knowledge sharing. For example, the organization can launch various training programs and team activities, implement evaluation, and provide rewards according to employees' performance.

5.3. Future Research Directions. This study contributes to the literature by shedding light on the crucial issue of a knowledge sharing mechanism in the workplace but has several limitations that point to future research directions. First, to simplify our model, we did not differentiate between organization types, while knowledge sharing shows different characteristics according to organization type. Future studies may take this issue into consideration to reflect the organizations' reality more accurately. Second, as knowledge is an important resource for an organization to obtain sustainable competitive advantage, the organization also needs to protect its knowledge from leaks, while encouraging employees to share knowledge adequately. This will thus help to further understand the knowledge sharing mechanism in the future investigations and discuss the boundary between knowledge sharing and knowledge protection. Finally, our study shows the results of knowledge sharing behaviors by constructing and simulating an evolutionary game model. Future empirical works would be helpful in verifying our conclusions.

\section{Appendix}

\section{A}

From equation (10), we derive

$$
\begin{aligned}
y^{*} & =\frac{\left(\beta_{1 P}^{-} \alpha_{1} k_{1 P}+c_{1}+c_{s}-\beta_{1 P}^{+} \alpha_{1} k_{1 P}-\beta_{1 G}^{+} \alpha_{1} k_{1 G}-f_{t}\right)}{\left\{\Gamma_{1}\left[\alpha_{1}\left(k_{1 P}+k_{1 G}\right)+\alpha_{2}\left(k_{2 P}+k_{2 G}\right)\right]-\tau_{1 P} \alpha_{2} k_{2 P}-\tau_{1 G} \alpha_{2} k_{2 G}+c_{s}+f_{s}\right\}} \\
& =\frac{-(3)}{(1)+c_{s}+f_{s}}=\frac{\left.-(5)-(1)-c_{s}-f_{s}\right)}{(1)+c_{s}+f_{s}}=1-\frac{\text { (5) }}{(1)+c_{s}+f_{s}}=\frac{V}{W} .
\end{aligned}
$$

We assume to obtain $0<y^{*}<1$ by following these two steps: (1) $y^{*}>0$ can be satisfied and (2) $y^{*}<1$ can be satisfied.

(1) In situation IX, the system can satisfy the following conditions: (1) $>0$, (2) $>0$, (3) $<0$, (4) $<0$, (5) $>0$, and (6) $>0$. As such, we can obtain $W>0$ (since (1) $\left.>0, c_{s}>0, f_{s}>0\right)$, and $V>0$ (since (3) $<0$ ), which means $y^{*}=V / W>0$.

(2) Furthermore, because (1) $>0$ and (5) $>0$, we derive $y^{*}=1-$ (5)/(1) $\left.+c_{s}+f_{s}\right)<1$.

In summary, it can be concluded that $0<y^{*}<1$.

By following the same derivation, we derive $0<x^{*}<1$.

\section{B}

From equations (14)-(21), we derive

$$
D_{g 1}^{\prime}=\frac{M}{Q}=\frac{-(5)}{Q}, D_{r 1}^{\prime}=\frac{N}{Q}=\frac{-(3)}{Q}, D_{g^{2}}^{\prime}=\frac{M^{\prime}}{Q^{\prime}}=\frac{-(6)}{Q^{\prime}}, D_{r 2}^{\prime}=\frac{N^{\prime}}{Q^{\prime}}=\frac{-(4)}{Q^{\prime}} \text {. }
$$

In situation IX, the system can satisfy the following conditions: (1) $>0$, (2) $>0$, (3) $<0$, (4) $<0$, (5) $>0$, and (6) $>0$. Additionally, to estimate whether $D_{g 1}{ }^{\prime}, D_{r 1}{ }^{\prime}$ and $D_{g 2}{ }^{\prime}, D_{r 2}{ }^{\prime}$ are positive or negative, we analyze them under two situations: (1) $Q<0, Q^{\prime}<0$; (2) $Q>0, Q^{\prime}>0$.

(1) When $Q<0, Q^{\prime}<0$, plus, (3) $<0$, (4) $<0$, (5) $>0$, (6) $>0$, it can be concluded that $D_{g 1}^{\prime}=-$ (5)/Q $>0$, $D_{r 1}^{\prime}=-$ (3) $/ Q<0, \quad$ and $\quad D_{g 2}^{\prime}=-(6 / Q>0$, $D_{r 2}^{\prime}=-(4) / Q<0$, which means this game is a chicken $(\mathrm{CH}$, also snowdrift $(\mathrm{SD})$, or hawk=dove $(\mathrm{HD}))$ game and has an internal (polymorphic) equilibrium.

(2) When $Q>0, Q^{\prime}>0$, plus, (3) $<0$, (4) $<0$, (5) $>0$, (6) $>0$, it can be concluded that $D_{g 1}^{\prime}=-$ (5) $/ Q<0$, $D_{r 1}^{\prime}=-\left(3 / Q>0, \quad\right.$ and $\quad D_{g 2}^{\prime}=-$ (6) $/ Q<0$, $D_{r 2}^{\prime}=-(4) / Q>0$, which means this game is a stag hunt $(\mathrm{SH})$ game that is bistable, and internal equilibrium $\left(x^{*}, y^{*}\right)$ falls into region $1>x^{*}>0$, and $1>y^{*}>0$. That is, $\{\mathrm{NKS}, \mathrm{NKS}\}$ and $\{\mathrm{KS}, \mathrm{KS}\}$ could both be the evolutionary stable strategy sets; therefore, the two players either both choose NKS or KS in the evolutionary stable state. Moreover, situation (2) is the focus of our research. As such, it can be concluded that the system needs to satisfy $Q>0, Q^{\prime}>0$. Then, we derive equations (B.2) and (B.3) as follows:

$$
\begin{aligned}
f_{t}> & -\left\{\Gamma_{1}\left[\alpha_{1}\left(k_{1 P}+k_{1 G}\right)+\alpha_{2}\left(k_{2 P}+k_{2 G}\right)\right]\right. \\
& \left.+\beta_{1 P}^{+} \alpha_{1} k_{1 P}+\beta_{1 G}^{+} \alpha_{1} k_{1 G}-\beta_{1 P}^{-} \alpha_{1} k_{1 P}-c_{1}\right\}, \\
f_{t}> & -\left\{\Gamma_{2}\left[\alpha_{1}\left(k_{1 P}+k_{1 G}\right)+\alpha_{2}\left(k_{2 P}+k_{2 G}\right)\right]\right. \\
+ & \left.\beta_{2 P}^{+} \alpha_{2} k_{2 P}+\beta_{2 G}^{+} \alpha_{2} k_{2 G}-\beta_{2 P}^{-} \alpha_{2} k_{2 P}-c_{2}\right\} .
\end{aligned}
$$

In summary, in situation IX, we can obtain $D_{g 1}{ }^{\prime}<0, D_{r 1}{ }^{\prime}>0$, and $D_{g 2}{ }^{\prime}<0, D_{r 2}{ }^{\prime}>0$ under the conditions of equations (B.2) and (B.3).

\section{Data Availability}

All data, models, and code generated or used during the study are included within the article. 


\section{Conflicts of Interest}

The authors declare no conflicts of interest.

\section{Authors' Contributions}

F. Song was responsible for the conceptualization, methodology, formal analysis, data curation, project administration, visualization, funding acquisition, writing the original draft preparation, and revising the manuscript. $\mathrm{X}$.W. Hu was involved in the funding acquisition, writing, reviewing, and editing; J.F. Li collected the resources; M.A. Watson wrote, reviewed, and edited the manuscript.

\section{Acknowledgments}

The authors are grateful to Yingxiu Zhao, and Editage, a brand of Cactus Communications, for their suggestions and proofreading. This work was funded by the Chinese National Funding of Natural Sciences, grant no. 42061033; General Project of State Ethnic Affair Commission of China, grant number 2020-GMB-028; Scientific Research and Innovation Team Project of Qinghai Nationalities University, China, grant no. 2019KYCXTD033; and Talents Project of Qinghai Nationalities University in 2021, China, grant no. $2021 X J G 01$.

\section{References}

[1] S. Kim and H. Lee, "The impact of organizational context and information technology on employee knowledge-sharing capabilities," Public Administration Review, vol. 66, no. 3, pp. 370-385, 2006.

[2] Z. Zhang, F. Song, and Z. Song, "Promoting knowledge sharing in the workplace: punishment v. reward," Solitons \& Fractals, vol. 10, 2019.

[3] R. McDermott and C. O’Dell, "Overcoming cultural barriers to sharing knowledge," Journal of Knowledge Management, vol. 5, no. 1, pp. 76-85, 2001.

[4] G. W. Bock, R. W. Zmud, Y. G. Kim, and F. Lee, "Behavioral intention formation in knowledge sharing: examining the roles of extrinsic motivators, social-psychological forces, and organizational climate," MIS Quarterly, vol. 29, no. 1, pp. 87-111, 2005.

[5] M. Gibbert and H. Krause, Practice Exchange in a Best Practice Marketplace in Knowledge Management Case Book: Siemens Best practices, Publics Corporate Publishing, Erlangen, Germany, 2002.

[6] M. Santos, "The evolution of anti-social rewarding and its countermeasures in public goods games," Proceedings of the Royal Society B: Biological Sciences, vol. 282, no. 1798, 2015.

[7] H. Gintis, "Strong reciprocity and human sociality," Journal of Theoretical Biology, vol. 206, no. 2, pp. 169-179, 2000.

[8] C. Bendersky and N. A. Hays, "Status conflict in groups," Organization Science, vol. 23, no. 2, pp. 323-340, 2012.

[9] P. H. Gray, "The impact of knowledge repositories on power and control in the workplace," Information Technology \& People, vol. 14, no. 4, pp. 368-384, 2001.

[10] B. Kogut and U. Zander, "Knowledge of the firm, combinative capabilities, and the replication of technology," Organization Science, vol. 3, no. 3, pp. 383-397, 1992.
[11] J. Park, H. Chae, and J. N. Choi, "The need for status as a hidden motive of knowledge-sharing behavior: an application of costly signaling theory," Human Performance, vol. 30, no. 1, pp. 1-17, 2017.

[12] S. Wang and R. A. Noe, "Knowledge sharing: a review and directions for future research," Human Resource Management Review, vol. 20, no. 2, pp. 115-131, 2010.

[13] B. Renzl, "Trust in management and knowledge sharing: the mediating effects of fear and knowledge documentation," Omega, vol. 36, no. 2, pp. 206-220, 2008.

[14] F. W. Marlowe, "Hadza cooperation," Human Nature, vol. 20, no. 4, pp. 417-430, 2009.

[15] E. Fehr and S. Gächter, "Fairness and retaliation: the economics of reciprocity," Journal of Economic Perspectives, vol. 14, no. 3, pp. 159-182, 2000.

[16] E. Fehr and S. Gächter, "Altruistic punishment in humans," Nature, vol. 415, no. 6868, pp. 137-140, 2002.

[17] K. M. Brethel-Haurwitz, S. A. Stoycos, E. M. Cardinale et al., "Is costly punishment altruistic? Exploring rejection of unfair offers in the Ultimatum Game in real-world altruists," Scientific Reports, vol. 6, no. 1, p. 18974, 2016.

[18] R. H. Thaler, “Anomalies: the ultimatum game," Journal of Economic Perspectives, vol. 2, no. 4, pp. 195-206, 1988.

[19] J. Gale, K. G. Binmore, and L. Samuelson, "Learning to be imperfect: the ultimatum game," Games and Economic Behavior, vol. 8, no. 1, pp. 56-90, 1995.

[20] E. Fehr and U. Fischbacher, "The nature of human altruism," Nature, vol. 425, no. 6960, pp. 785-791, 2003.

[21] R. H. Thaler and C. R. Sunstein, Nudge: Improving Decisions about Health, Wealth and happiness, Yale University Press, London, UK, 2008.

[22] R. Boyd, H. Gintis, S. Bowles, and P. J. Richerson, "The evolution of altruistic punishment," in Proceedings of the National Academy of Sciences, vol. 100, no. 6, pp. 3531-3535, 2003.

[23] J. Henrich, R. McElreath, A. Barr et al., "Costly punishment across human societies," Science, vol. 312, no. 5781, pp. 1767-1770, 2006.

[24] Y. Zhou, P. Jiao, and Q. Zhang, "Second-party and third-party punishment in a public goods experiment," Applied Economics Letters, vol. 24, no. 1, pp. 54-57, 2017.

[25] E. Fehr and U. Fischbacher, "Third-party punishment and social norms," Evolution and Human Behavior, vol. 25, no. 2, pp. 63-87, 2004.

[26] A. Leibbrandt and R. López-Pérez, "An exploration of third and second party punishment in ten simple games," Journal of Economic Behavior \& Organization, vol. 84, no. 3, pp. 753766, 2012.

[27] C. D. Batson, C. L. Kennedy, L.-A. Nord et al., "Anger at unfairness: is it moral outrage?" European Journal of Social Psychology, vol. 37, no. 6, pp. 1272-1285, 2007.

[28] J. Bendor and P. Swistak, "The evolution of norms," American Journal of Sociology, vol. 106, no. 6, pp. 1493-1545, 2001.

[29] C. H. Loch, B. A. Huberman, and S. Stout, "Status competition and performance in work groups," Journal of Economic Behavior \& Organization, vol. 43, no. 1, pp. 35-55, 2000.

[30] J. H. Barkow, The Transition from Primate Dominance to Human Self Esteem, University of Toronto Press, Toronto, TO, USA, 1989.

[31] C. Anderson, J. A. D. Hildreth, and L. Howland, "Is the desire for status a fundamental human motive? A review of the empirical literature," Psychological Bulletin, vol. 141, no. 3, pp. 574-601, 2015. 
[32] E. Von Hippel, Cooperation between Rivals: Informal KnowHow Trading, Industrial dynamics Springer, Dordrecht, MA, USA, 1989.

[33] C. Ridgeway, "The social construction of status value: gender and other nominal characteristics," Social Forces, vol. 70, no. 2, pp. 367-386, 1991.

[34] E. V. Leeuwen and S. Tauber, "Demonstrating knowledge: the effects of group status on outgroup helping," Journal of Experimental Social Psychology, vol. 47, no. 1, pp. 147-156, 2011.

[35] F. Gino and J. D. Margolis, "Bringing ethics into focus: how regulatory focus and risk preferences influence (Un)ethical behavior," Organizational Behavior \& Human Decision Processes, vol. 115, no. 2, pp. 145-156, 2010.

[36] R. Kark and D. Van Dijk, "Motivation to lead, motivation to follow: the role of the self-regulatory focus in leadership processes," Academy of Management Review, vol. 32, no. 2, pp. 500-528, 2007.

[37] W. Wei and Z. Y. Huo, "Antecedents and intervention mechanisms: a multi-level study of R \& D team's knowledge hiding behavior," Journal of Knowledge Management, vol. 20, no. 5, pp. 880-897, 2016.

[38] Y. W. Rhee and J. N. Choi, "Knowledge management behavior and individual creativity: goal orientations as antecedents and in-group social status as moderating contingency," Journal of Organizational Behavior, vol. 38, no. 6, pp. 813-832, 2016.

[39] A. P. Carter, "Knowhow trading as economic exchange," Research Policy, vol. 18, no. 3, pp. 155-163, 1989.

[40] J. Nolan and C. Rowley, "Whither guanxi and social networks in China? A review of theory and practice," Asia Pacific Business Review, vol. 26, no. 2, pp. 113-123, 2020.

[41] D. Y. Lee and P. L. Dawes, "Guanxi, trust, and long-term orientation in Chinese business markets," Journal of International Marketing, vol. 13, no. 2, pp. 28-56, 2005.

[42] E. W. K. Tsang, "Can guanxi be a source of sustained competitive advantage for doing business in China?" Academy of Management Perspectives, vol. 12, no. 2, pp. 64-73, 1998.

[43] G. Thomas, D. Guthrie, and D. Wank, Social Connections in China, Cambridge University Press, Cambridge, UK, 2002.

[44] X. T. Fei, From the Soil, University of California Press, Berkeley, CA, USA, 1992.

[45] J. B. Jacobs, "A preliminary model of particularistic ties in Chinese political alliances: kan-ch'ing and kuan-hsi in a rural Taiwanese township," The China Quarterly, vol. 78, pp. 237273, 1979.

[46] K.-K. Hwang, "Face and favor: the Chinese power game," American Journal of Sociology, vol. 92, no. 4, pp. 944-974, 1987.

[47] C. Chiao, "Guanxi: a preliminary conceptualization," The Sinicization of Social and Behavioral Science Research in China, vol. 20, pp. 345-360, 1982.

[48] K. S. Yang, Gifts, Favors, and Banquets: The Art of Social Relationships in China, Cornell University Press, New York, NY, USA, 1994.

[49] R. H. Lin and J. Mi, "Knowledge sharing and indirect reciprocity: research based on computational experiments," Operations Research and Management Science, vol. 09, pp. 161-169, 2017.

[50] Y. Liu, T. Chen, and Y. Wang, "Sustainable cooperation in Village Opera based on the public goods game," Chaos, Solitons \& Fractals, vol. 103, pp. 213-219, 2017.

[51] V. L. Smith, "Constructivist and ecological rationality in economics," American Economic Review, vol. 93, no. 3, pp. 465-508, 2003.
[52] Q. Li and Y. Kang, "Knowledge sharing willingness and leakage risk: an evolutional game model," Sustainability, vol. 11, no. 3, p. 596, 2019.

[53] R. H. Wang, Y. B. Lv, and M. Duan, "Evolutionary game of inter-firm knowledge sharing in innovation cluster," Evolving Systems, vol. 8, no. 2, pp. 1-13, 2016.

[54] M. Ipe, "Knowledge sharing in organizations: a conceptual framework," Human Resource Development Review, vol. 2, no. 4, pp. 337-359, 2003.

[55] K. M. Andrews and B. L. Delahaye, "Influences on knowledge processes in organizational learning: the psychological filter [J]," Journal of Management Studies, vol. 37, no. 6, pp. 2322-2380, 2000.

[56] D. Friedman, "Evolutionary games in economics," Econometrica, vol. 59, no. 3, pp. 637-666, 1991.

[57] J. Tanimoto and H. Sagara, "Relationship between dilemma occurrence and the existence of a weakly dominant strategy in a two-player symmetric game," BioSystems, vol. 90, no. 1, pp. 105-114, 2007.

[58] Z. Wang, S. Kokubo, M. Jusup, and J. Tanimoto, "Universal scaling for the dilemma strength in evolutionary games," Physics of Life Reviews, vol. 14, pp. 1-30, 2015.

[59] H. Ito and J. Tanimoto, "Scaling the phase-planes of social dilemma strengths shows game-class changes in the five rules governing the evolution of cooperation," Royal Society Open Science, vol. 5, no. 10, pp. 1-9, 2018. 